\title{
Diurnal dynamics of the
}

\section{Arabidopsis rosette proteome and phosphoproteome}

\section{Journal Article}

\section{Author(s):}

Uhrig, R. Glen; Echevarria-Zomeno, Sira; Schläpfer, Pascal (D); Grossmann, Jonas; Roschitzki, Bernd; Koerber, Niklas; Fiorani, Fabio; Gruissem, Wilhelm (1)

Publication date:

2021-03

Permanent link:

https://doi.org/10.3929/ethz-b-000473259

\section{Rights / license:}

Creative Commons Attribution-NonCommercial-NoDerivatives 4.0 International

\section{Originally published in:}

Plant, Cell \& Environment 44(3), https://doi.org/10.1111/pce.13969 


\title{
Diurnal dynamics of the Arabidopsis rosette proteome and phosphoproteome
}

\author{
R. Glen Uhrig ${ }^{1,2}$ | Sira Echevarría-Zomeño ${ }^{1}$ | Pascal Schlapfer ${ }^{1}$ | \\ Jonas Grossmann $^{3}$ | Bernd Roschitzki ${ }^{3}$ | Niklas Koerber ${ }^{4}$ | Fabio Fiorani ${ }^{4}$ | \\ Wilhelm Gruissem ${ }^{1,5}$
}

\footnotetext{
${ }^{1}$ Department of Biology, Institute of Molecular Plant Biology, ETH Zurich, Zurich, Switzerland

${ }^{2}$ Department of Biological Sciences, University of Alberta, Edmonton, Alberta, Canada

${ }^{3}$ Functional Genomics Center Zurich, University of Zurich, Zurich, Switzerland

${ }^{4}$ Institute of Bio- and Geosciences, IBG-2: Plant Sciences, Forschungszentrum Jülich $\mathrm{GmbH}$, Jülich, Germany

${ }^{5}$ Institute of Biotechnology, National Chung Hsing University, Taichung, Taiwan

\section{Correspondence}

Wilhelm Gruissem, Department of Biology, ETH Zurich, Universitätstrasse 28092 Zurich Switzerland.13969

E-mail:wgruissem@ethz.ch

Funding information

Ministry of Education (Taiwan); Helmholtz Association; European Commission (FP7-KBBE-2009-3); TiMet - Linking the Clock to Metabolism, Grant/Award Number: Grant Agreement 245143
}

\begin{abstract}
Plant growth depends on the diurnal regulation of cellular processes, but it is not well understood if and how transcriptional regulation controls diurnal fluctuations at the protein level. Here, we report a high-resolution Arabidopsis thaliana (Arabidopsis) leaf rosette proteome acquired over a $12 \mathrm{hr}$ light:12 hr dark diurnal cycle and the phosphoproteome immediately before and after the light-to-dark and dark-to-light transitions. We quantified nearly 5,000 proteins and 800 phosphoproteins, of which 288 fluctuated in their abundance and 226 fluctuated in their phosphorylation status. Of the phosphoproteins, $60 \%$ were quantified for changes in protein abundance. This revealed six proteins involved in nitrogen and hormone metabolism that had concurrent changes in both protein abundance and phosphorylation status. The diurnal proteome and phosphoproteome changes involve proteins in key cellular processes, including protein translation, light perception, photosynthesis, metabolism and transport. The phosphoproteome at the light-dark transitions revealed the dynamics at phosphorylation sites in either anticipation of or response to a change in light regime. Phosphorylation site motif analyses implicate casein kinase II and calcium/ calmodulin-dependent kinases among the primary light-dark transition kinases. The comparative analysis of the diurnal proteome and diurnal and circadian transcriptome established how mRNA and protein accumulation intersect in leaves during the diurnal cycle of the plant.
\end{abstract}

\section{KEYWORDS}

Arabidopsis thaliana, diurnal cycle, quantitative proteomics
R. Glen Uhrig and Sira Echevarría-Zomeño contributed equally to this study and should be considered joint first authors.

\section{1 | INTRODUCTION}

Plant growth and biomass production are direct functions of the diurnal cellular carbon balance, which is regulated by a combination of light responses and the circadian clock. Light responses are triggered 
by a change in regime (i.e., presence or absence of light), while the circadian clock is comprised of transcriptional regulators that operate in anticipation of a change (e.g., transition from light to dark) and whose activities span the $24 \mathrm{hr}$ (hr) photoperiod (Nohales \& Kay, 2016; Oakenfull \& Davis, 2017; Seluzicki, Burko, \& Chory, 2017). The core clock transcriptional regulators include CCA1/LHY, PRR5, PRR7 and PRR9, which form the morning loop, and TOC1, ELF3, ELF4 and LUX, which form the evening loop (Flis et al., 2015; Staiger, Shin Johansson, \& Davis, 2013). More than 30\% of all Arabidopsis genes are regulated by the circadian clock at the transcript level (Blasing et al., 2005; Covington, Maloof, Straume, Kay, \& Harmer, 2008). However, less is known about how the resulting diurnal transcriptome relates to protein abundance (Abraham et al., 2016; Choudhary, Nomura, Wang, Nakagami, \& Somers, 2015; Graf et al., 2017) and posttranslational protein modifications (Choudhary et al., 2015; Uhrig, Schlapfer, Roschitzki, Hirsch-Hoffmann, \& Gruissem, 2019), both of which may also affect protein function at light-dark transitions and throughout the diurnal cycle. Transcript and protein abundance changes are often disconnected because changes in transcript levels show no corresponding change in protein abundance (Baerenfaller et al., 2012; Seaton et al., 2018). For example, this disconnect was found in the circadian clock mutants CCA1/LHY, PRR7/PRR9, TOC1 and GI (Graf et al., 2017) and for the variability in the timing of peak transcript and protein levels (translational coincidence) as a function of the photoperiod-dependent coordination between transcriptome and proteome changes (Seaton et al., 2018). Variable delays between peak transcript and protein abundance have implicated post-transcriptional regulation (e.g., splicing), translational regulation (e.g., translation rate) as well as post-translational regulation (e.g., protein phosphorylation) as possible mechanisms to explain the temporal differences in RNA and protein abundance. More recent studies of plant protein-level regulation have also found extensive variability in protein turnover ( $\mathrm{Li}$, Nelson, et al., 2017; Seaton et al., 2018), which adds further regulatory complexity because conventional quantitative proteome workflows cannot easily account for protein turnover. Although transcript and protein synthesis, stability and turnover all contribute to the coordination of transcript and protein abundance, how these mechanisms are integrated is currently not well understood. Insights into this regulatory complexity requires protein-level time-course experimentation and, in particular, an understanding of protein post-translational modifications (PTMs). To address this, we undertook a large-scale quantitative proteomics approach to determine the extent of diurnal protein abundance and/or phosphorylation changes in Arabidopsis rosette proteins over a $24 \mathrm{hr}$ photoperiod.

Reversible protein phosphorylation is the most abundant PTM in eukaryotes (Adam \& Hunter, 2018; Rao, Thelen, \& Miernyk, 2014) that modulates more than $70 \%$ of all cellular processes (Olsen et al., 2006), including the circadian clock itself (Robles, Humphrey, \& Mann, 2017). This is likely similar in land plants, which have a larger number of protein kinases (PKs) than humans (Lehti-Shiu \& Shiu, 2012; Manning, Whyte, Martinez, Hunter, \& Sudarsanam, 2002; Zulawski, Schulze, Braginets, Hartmann, \& Schulze, 2014). Conversely, plants and humans have a comparable number of protein phosphatases (Kerk, Templeton, \& Moorhead, 2008), which in plants also require association with regulatory subunits to achieve their specificity (Moorhead et al., 2008; Uhrig, Labandera, \& Moorhead, 2013).

In plants, diurnal protein phosphorylation is regulated either in response to light, by the circadian clock (Choudhary et al., 2015), or both (Uhrig et al., 2019), while the clock itself is regulated by phosphorylation (Kusakina \& Dodd, 2012; Uehara et al., 2019). Recent studies of the circadian phosphoproteome combining the analysis of a free-running cycle and the circadian clock mutants elf4 (Choudhary et al., 2015) or CCA1-OX over-expression (Krahmer et al., 2019) have revealed temporally modified phosphorylation sites related to casein kinase II (CKII) and sucrose non-fermenting kinase 1 (SnRK1). SnRKs are likely involved in the regulation of the circadian phosphoproteome because the transcription of genes encoding multiple SnRK and calcinuerin $\mathrm{B}$-like (CBL) interacting kinases (CIPK) was mis-regulated in the Arabidopsis circadian clock mutants cca1/lhy1, prr7prr9, toc1 and gi201 mutants at end-of-day (ED) and end-of-night (EN) (Graf et al., 2017). Similarly, studies quantifying changes in the phosphoproteome at ED and EN in Arabidopsis rosette leaves, roots, flowers, siliques and seedlings have revealed a large number of diurnally changing phosphorylation events corresponding to diverse PK motifs (Reiland et al., 2009; Uhrig et al., 2019).

Considering the marked physiological and metabolic changes occurring at the light-dark (L-D) and dark-light (D-L) transitions (Annunziata et al., 2018; Gibon et al., 2009; Usadel et al., 2008), we performed a quantitative phosphoproteome analysis of proteins that are phosphorylated immediately before and after the L-D and D-L transitions during a $12 \mathrm{hr}$ light:12 hr dark photoperiod and asked how these phosphorylation events intersect with changes in protein abundance. Together, our systems-level quantitative analysis of the Arabidopsis thaliana rosette proteome and phosphoproteome over a $24 \mathrm{hr}$ photoperiod provides new insights into diurnal protein and phosphorylation regulation.

\section{2 | MATERIALS AND METHODS}

Arabidopsis Col-0 wild-type plants were grown at the Forschungszentrum Jülich (Germany) in an environmentally controlled chamber (GrowScreen Chamber; https://eppn2020.plant-phenotyping. eu/EPPN2020_installations\#/tool/30; Barboza-Barquero et al., 2015) under a $12 \mathrm{hr}$ light:12 hr dark photoperiod and controlled conditions as described in Baerenfaller et al. (2012), including air temperature of $21^{\circ} \mathrm{C}$ during the day and $20^{\circ} \mathrm{C}$ during the night, air humidity of $70 \%$, and an incident light intensity of $\sim 220 \mathrm{mmol} / \mathrm{m}^{2} / \mathrm{s}$ at the plant level. Whole rosettes were harvested at 31 days after sowing (DAS) prior to flowering. Four whole rosettes were pooled into one sample and four biological replicates were collected at each time point except for ZT1, 3, 59 and 23 , which had only three biological replicas for proteome analysis and AL_10, which had only three replicas for phosphoproteome analysis. For total proteome analyses, samples were taken every $2 \mathrm{hr}$ during $24 \mathrm{hr}$, starting at Zeitgeber time 1 (ZT1, i.e., $1 \mathrm{hr}$ after lights turned on). For 
protein phosphorylation analyses, samples were $30 \mathrm{~min}$ before, $10 \mathrm{~min}$ after and $30 \mathrm{~min}$ after the L-D and D-L transitions. Samples were snapfrozen in liquid $\mathrm{N}_{2}$ and stored at $-80^{\circ} \mathrm{C}$ until protein extraction.

\section{1 | Proteome analysis}

\subsubsection{Extraction and digestion}

Samples were randomized before processing to avoid batch effects. Frozen rosettes were ground under liquid $\mathrm{N}_{2}$. Proteins were extracted from $100 \mathrm{mg}$ of frozen powder per sample by adding $150 \mu \mathrm{l}$ of extraction buffer (30 mM Tris- $\mathrm{HCl} \mathrm{pH} \mathrm{8.0,4 \%} \mathrm{SDS).} \mathrm{Tubes} \mathrm{were} \mathrm{incubated}$ in a shaker (Eppendorf) at $4^{\circ} \mathrm{C}$ at $1400 \mathrm{rpm}$ for $30 \mathrm{~min}$. Samples were centrifuged at $16000 \mathrm{~g}$ and $4^{\circ} \mathrm{C}$ for $30 \mathrm{~min}$ and the supernatant was transferred to a new tube. Protein concentration was estimated based on Bradford (Bradford, 1976) using the Bio-Rad Protein Assay reagent. Subsequently, dithiothreitol (DTT) was added to a final concentration of $50 \mathrm{mM}$ and proteins were reduced for $30 \mathrm{~min}$ on ice. For digestion, $140 \mu \mathrm{g}$ of proteins were processed following the filter assisted sample preparation method (Wisniewski, Zougman, Nagaraj, \& Mann, 2009). Peptides were desalted using SPE C18 columns (Finisterre) and dried down in a SpeedVac concentrator.

\subsection{2 | Peptide fractionation}

To increase proteome coverage, peptide samples were fractionated by hydrophilic interaction chromatography on an Agilent 1200 series high performance liquid chromatography system with a YMC-Pack Polyamine II $250 \times 3.0 \mathrm{~mm}$ size column with $5 \mu \mathrm{m}$ particle size and $120 \AA$ A pore size. Samples were dissolved in $100 \mu$ l Buffer A (75\% acetonitrile (ACN), $8 \mathrm{mM} \mathrm{KH}_{2} \mathrm{PO}_{4}, \mathrm{pH} 4.5$ ) and separated with Buffer $\mathrm{B}$ ( $5 \% \mathrm{ACN}, 100 \mathrm{mM} \mathrm{KH}_{2} \mathrm{PO}_{4}, \mathrm{pH} 4.5$ ) at a flow rate of $500 \mu \mathrm{l} / \mathrm{min}$ with the following gradient: $0-7.5 \mathrm{~min}, 0 \% \mathrm{~B} ; 7.5-37.5 \mathrm{~min}, 0-50 \% \mathrm{~B}$; 37.5-42.5 min, $50-100 \% \mathrm{~B} ; 42.5-47.5 \mathrm{~min}, 100 \%$ B. Following separation, the column was washed with $100 \%$ buffer $A$ and re-equilibrated for $60 \mathrm{~min}$. For each sample, the 27 automatically collected fractions were pooled into five fractions that were subsequently dried down in a SpeedVac concentrator. Each sample was then dissolved in $200 \mu \mathrm{l}$ of $3 \%$ ACN, 0.1\% TFA, desalted on SPE C18 columns (Finisterre) and again dried in a SpeedVac concentrator.

\subsection{3 | LC-MS analysis}

Mass spectrometry queues were arranged to process comparable fractions in the same batch, with sample order randomized within each batch. Peptide samples were dissolved in $20 \mu \mathrm{l} 3 \% \mathrm{ACN}, 0.1 \%$ FA and spiked with internal retention timestandards (Biognosys) for chromatography quality control. LC-MS/MS shotgun analyses were performed on a Thermo Orbitrap Fusion instrument coupled to an Eksigent NanoLC Ultra (Sciex). Samples were separated on a self- packed reverse-phase column $(75 \mu \mathrm{m} \times 150 \mathrm{~mm})$ with $\mathrm{C} 18$ material (ReproSil-Pur, C18, $120 \AA$, AQ, $1.9 \mu \mathrm{m}$, Dr. Maisch GmbH). The column was equilibrated with $100 \%$ solvent $A$ (0.1\% FA in water). Peptides were eluted using the following gradient of solvent $B(0.1 \%$ FA in $\mathrm{ACN}$ ) at a flow rate of $0.3 \mu \mathrm{l} / \mathrm{min}: 0-50 \mathrm{~min}: 3-25 \% \mathrm{~B}$, 50-60 min: $25-35 \%$ B, $60-70$ min: $35-97 \%$ B, $70-80$ min: $97 \%$ B, 80-85 min: $2 \%$ B. Mass spectra were acquired in a data-dependent manner. All precursor signals were recorded in the Orbitrap using quadrupole transmission in the mass range of $300-1,500 \mathrm{~m} / \mathrm{z}$. Spectra were recorded with a resolution of 120000 (FWHM) at $200 \mathrm{~m} / \mathrm{z}$, a target value of $4 \mathrm{e} 5$ and the maximum cycle time set to $3 \mathrm{~s}$. Datadependent MS/MS were recorded in the linear ion trap using quadrupole isolation with a window of $1.6 \mathrm{Da}$ and higher-energy collisional dissociation (HCD) fragmentation with $30 \%$ fragmentation energy. The ion trap was operated in rapid scan mode with a target value of 1E4 and a maximum injection time of $250 \mathrm{~ms}$. Precursor signals were selected for fragmentation with a charge state from +2 to +7 and a signal intensity of at least $5 \mathrm{e} 3$. A dynamic exclusion list was used for $30 \mathrm{~s}$ and maximum parallelizing ion injections was activated. The mass spectrometry proteomics data were handled using the local laboratory information management system (LIMS) (Türker et al., 2010).

\section{2 | Phosphoproteome analysis}

\subsection{1 $\quad$ Extraction}

Whole rosette tissue from each time point was harvested and ground under liquid $\mathrm{N}_{2}$. From each biological replicate, $200 \mathrm{mg}$ of ground leaf material was weighed out under liquid $\mathrm{N}_{2}$. In addition to each biological replicate, $200 \mathrm{mg}$ of samples containing equal weighted parts of each biological replicate and time-point were created as a reference sample (gold standard) for downstream dimethyl labelling. All proteins were extracted in a $250 \mu$ solution of $50 \mathrm{mM}$ HEPES pH 8.0, $6 \mathrm{M}$ urea, $2 \mathrm{M}$ thiourea, $100 \mathrm{mM} \mathrm{NaCl}, 10 \mathrm{mM}$ EDTA, $2 \mathrm{mM} \mathrm{NaOV}, 5 \mathrm{mM} \mathrm{NaF}$, $50 \mu \mathrm{g} / \mathrm{ml}$ PhosSTOP (Roche). Samples were shaken at room temperature for $30 \mathrm{~min}$ at $1000 \mathrm{~g}$ with vortexing every $10 \mathrm{~min}$. Extracts were then brought to $\mathrm{pH} 8.0$ using triethylammonium bicarbonate (TEAB). Protein extracts were then reduced for $30 \mathrm{~min}$ with $10 \mathrm{mM}$ DTT, followed by alkylation with $30 \mathrm{mM}$ iodoacetamide for $1 \mathrm{hr}$. Extracts were clarified to separate soluble and insoluble fractions. The insoluble fraction was re-suspended in $300 \mu \mathrm{l} 60: 40$ buffer containing $60 \%$ $\mathrm{MeOH}: 40 \% 50 \mathrm{mM}$ TEAB pH 8.0 followed by shaking at $1000 \mathrm{rpm}$ (Eppendorf tabletop) for $2.5 \mathrm{hr}$. The protein concentration of the soluble fraction was then measured using the Bradford protein assay (Bradford, 1976). An amount of $1 \mathrm{mg}$ of soluble protein from each sample was then diluted with 1 vol. of $50 \mathrm{mM}$ TEAB and then water was added to a total volume of $1.2 \mathrm{ml}$ and a final urea/thiourea concentration of $1.2 \mathrm{M}$. The soluble fraction was then digested for $20 \mathrm{hr}$ at $37^{\circ} \mathrm{C}$ using a 1:50 ratio of trypsin (Promega) to extracted protein while gently shaking. Each insoluble fraction was digested by $0.5 \mu \mathrm{g}$ chymotrypsin and $1 \mu \mathrm{g}$ trypsin at $37^{\circ} \mathrm{C}$ for $20 \mathrm{hr}$ shaking at $600 \mathrm{rpm}$ (Eppendorf 
tabletop). Digestion reactions were stopped using TFA to a final concentration of $0.5 \%$. The insoluble fractions were centrifuged for $10 \mathrm{~min}$ at $20000 \mathrm{~g}$ at room temperature and the supernatant removed. The supernatant was then dried and re-suspended in desalting buffer comprised of $3 \%$ ACN/0.1\% TFA. The soluble fraction and the supernatant from the insoluble fraction were desalted using SPE C18 columns (Finisterre) and dried in a SpeedVac concentrator.

\subsection{2 | Dimethyl labeling and phosphopeptide enrichment}

Total peptide fractions from each experimental (light label) and goldstandard (heavy label) sample were labelled according to Boersema et al. (Boersema, Raijmakers, Lemeer, Mohammed, \& Heck, 2009). Heavy and light samples were then mixed 1:1 and desalted prior to phosphopeptide enrichment using $\mathrm{TiO}_{2}$. Phosphopeptide enrichment was performed using $\mathrm{TiO}_{2}$ heavy and light dimethyl-labelled phosphopeptides as previously described (Zhou et al., 2011).

\subsection{3 | LC-MS}

Phosphorylated peptide samples were analysed using a Q Exactive Orbitrap mass spectrometer (Thermo Scientific). Dissolved samples were injected using an Easy-nLC 1,000 system (Thermo Scientific) and separated on a self-made reverse-phase column $(75 \mu \mathrm{m} \times 150 \mathrm{~mm})$ packed with C18 material (ReproSil-Pur, C18, $120 \AA ., A Q, 1.9 \mu \mathrm{m}$, Dr. Maisch $\mathrm{GmbH}$ ). The column was equilibrated with $100 \%$ solvent $A$ (0.1\% formic acid [FA] in water). Peptides were eluted using the following gradient of solvent $B(0.1 \% \mathrm{FA}$ in $\mathrm{ACN})$ : 0-120 min, $0-35 \% \mathrm{~B}$, $120-122 \mathrm{~min}, 35-95 \% \mathrm{~B}$ at a flow rate of $0.3 \mu \mathrm{l} / \mathrm{min}$. High accuracy mass spectra were acquired in data-depended acquisition mode. All precursor signals were recorded in a mass range of $300-1700 \mathrm{~m} / \mathrm{z}$ and a resolution of 70000 at $200 \mathrm{~m} / \mathrm{z}$. The maximum accumulation time for a target value of $3 \mathrm{e} 6$ was set to $120 \mathrm{~ms}$. Up to 12 datadependent MS/MS were recorded using quadrupole isolation with a window of $2 \mathrm{Da}$ and $\mathrm{HCD}$ fragmentation with $28 \%$ fragmentation energy. A target value of 1 e6 was set for MS/MS using a maximum injection time of $250 \mathrm{~ms}$ and a resolution of 70,000 at $200 \mathrm{~m} / \mathrm{z}$. Precursor signals were selected for fragmentation with charge states from +2 to +7 and a signal intensity of at least $1 \mathrm{e} 5$. All precursor signals selected for MS/MS were dynamically excluded for $30 \mathrm{~s}$.

\section{3 | Quantitative analysis and bioinformatics}

\section{\begin{tabular}{l|l} 
2.3.1 & Total proteome
\end{tabular}}

Label-free precursor (MS1) intensity-based quantification was performed using Progenesis QI for Proteomics (version 2.1, www. nonlinear.com) to quantify total proteome changes. Briefly, for each individual fraction, automatic alignment was reviewed and manually adjusted before normalization. From each Progenesis peptide ion (default sensitivity in peak picking), a maximum of the top five tandem mass spectra per peptide ion were exported as a Mascot generic file ( ${ }^{*}$.mgf) using charge deconvolution and deisotoping option and a maximum number of 200 peaks per MS/MS. Searches were done in Mascot 2.4.1 (Matrix Science) against a decoyed (reversed) Arabidopsis protein database from TAIR (release TAIR10) concatenated with a collection of 261 known mass spectrometry contaminants. Precursor ion mass tolerance was set to $10 \mathrm{ppm}$ and the fragment ion mass tolerance was set to $0.6 \mathrm{Da}$. The following search parameters were used: trypsin digestion (1 missed cleavage allowed), fixed modifications of carbamidomethyl modified cysteine and variable modifications of oxidation of methionine, deamidation of asparagine and glutamine, and acetylation of protein N-terminal peptides. Mascot searches were imported into Scaffold 4.2.1 (Proteome Software). The following thresholds were applied: peptide FDR $\leq 5$, protein FDR $\leq 10,1$ minimum peptide. Spectrum reports were imported again into Progenesis. After this, individual fraction analyses were combined into the full quantitative Progenesis experiment. From this, quantitative peptide values were exported for further processing. Only peptides that could be unambiguously assigned to a single protein (gene model annotation) were kept for quantification. A Hi-4 strategy (Grossmann et al., 2010) was applied to obtain protein quantitative values. Proteins with two or more peptides assigned were considered as quantifiable. Following these criteria, the final protein level FDR was estimated at 0.013 .

\subsection{2 | Phosphoproteome}

Quantification of changes in identified phosphopeptides was performed using MaxQuant (version 1.3.0.5) with default settings and the following modifications: fixed peptide modification by carbamidomethylation of cysteines and variable peptide modifications by phosphorylation of serine, threonine, and tyrosine, and oxidation of methionine, and false discovery rate (FDR) tolerances of $\leq 0.05$ (protein) and $\leq 0.01$ (peptide). MaxQuant outputs were subsequently filtered for phosphopeptides with a phosphorylation site probability score $\geq 0.8$ and presence in at least 2 of 3 (AL_10) or 2 of 4 biological replicates and 2 of 3 time-points for each light transition.

\subsection{3 | Data analysis}

Significant fluctuations in protein abundance and phosphopeptides were determined using an ANOVA analysis: total proteome ( $p$ value $\leq .05$ and fold-change $[F C] \geq 1.5$ ) and phosphoproteome ( $p$ value $\leq .05)$. The significantly changing proteome was subjected to cluster analysis using GProX (Rigbolt, Vanselow, \& Blagoev, 2011). Six clusters were generated in an unsupervised clustering manner based on the fuzzy c-means algorithm. Significantly changing proteins and phosphoproteins were subjected to gene set enrichment analysis (GSEA) using the SetRank algorithm relative to the identified 
proteome and phosphoproteome, respectively (Simillion, Liechti, Lischer, loannidis, \& Bruggmann, 2017). Enrichment was calculated for all the available databases included in the SetRank R package. Only terms with a size $\geq 2$ were considered (gene set size $\geq 2$ ). For each protein cluster, a SetRank corrected $p$ value $\leq .01$ was applied as threshold. For phosphoproteins changing at the L-D or D-L transition, a SetRank corrected $p$ value $\leq .01$ and an FDR $\leq 0.05$ were applied. To test for significantly non-changing proteins at the transitions to light, (i.e., at dawn, ZT23 to ZT1, and dusk, ZT11 to ZT13), a TOST equivalence test (equivalence $R$ package) was applied with an $\varepsilon=0.4$. Significance threshold was $p$ value $\leq .05$. The mass spectrometry proteomics data have been deposited to the ProteomeXchange Consortium via the PRIDE partner repository. Data are available via ProteomeXchange with identifier PXD007600.

\subsubsection{Additional analyses}

To compare protein and mRNA profiles, mRNA data generated by the Alison Smith laboratory were obtained from the Diurnal database (http://diurnal.mocklerlab.org; Mockler et al., 2007). For this, we restricted the analysis to the information from LDHH_SM and LDHH_ST. Data were standardized to plot both protein and mRNA data in the same graph. Predicted subcellular localization of all changing proteins and phosphoproteins was performed using the consensus subcellular localization predictor SUBAcon (suba3. plantenergy.uwa.edu.au) (Tanz et al., 2013). String DB network analyses were undertaken using both proteome and phosphoproteome data. String DB analyses were performed in Cytoscape using the String DB plugin stringApp (Szklarczyk et al., 2017). A minimum correlation coefficient of 0.5 was used along with a second layer of five additional nodes to anchor each network to better infer network connectedness.

\subsection{5 | JTK analyses}

tTo compare diurnal protein fluctuations to free running circadian clock fluctuations published by Krahmer et al. (2019); dataset PXD009230 available at ProteomeXchange), we performed an equivalent analysis using the JTK cycle to identify proteins cycling with 22 or $24 \mathrm{hr}$ period (Hughes, Hogenesch, \& Kornacker, 2010). The exact loading script JTK_analysis.zip is available upon request. JTK_cycle fits data of many entities (here protein abundances) to a cosine function model, and estimates a $p$ value for the accuracy of the model for every protein permutation of the dataset (resulting in the ADJ.P values). Further, it applies a Benjamini Hochberg correction for multiple testing resulting in q-values (resulting in the BH.Q values). The data were then used to produce Figure $3 b-d$. Proteins identified to fluctuate were normalized such that they fluctuate around a median of 0 with maximal amplitudes of 2. Transcriptome data from Diurnal DB (http://diurnal.mocklerlab. org; Mockler et al., 2007) were used to determine if the associated transcripts were also fluctuating, and if so, when. For this, we restricted the analysis to the information from LDHH_SM and LDHH_ST. To estimate a confidence interval for the relative expression or protein level errors, their relative levels were compared to the theoretical cosine function at the same timepoint. Based on all errors, irrespective of the exact timepoint, a 99\% confidence interval was computed.

\section{3 | RESULTS AND DISCUSSION}

\section{1 | Dynamics of the Arabidopsis diurnal proteome and phosphoproteome}

Using proteotypic peptides, we performed a label-free quantitative proteomics analysis of the diurnal proteome. Here, we identified 7,060 proteins, of which we were able to quantify 4,762 with two and more proteotypic peptides over the $24 \mathrm{hr}$ time-course (Figure S1; Table 1; Table S1). Statistical analysis showed that 288 of these proteins were significantly changing in abundance (ANOVA $p$ value $\leq .05$, FC > 1.5); Table 1; Table S2), suggesting that a portion ( 6\%) of the quantified proteome is dynamically regulated over the course of a day. Additionally, using a dimethyl-labelling approach, we identified a total of 2,298 phosphopeptides (Figure S1; Table S3), of which 1776 had a phosphorylation site probability score $\geq 0.8$. We were able to quantify 1,056 of these phosphopeptides (Table 1), which corresponded to a total of 1803 identified phosphorylation sites. Of these, 253 (14\%) represented newly identified phosphorylation sites when compared to the compendium of 79,334 known phosphorylation sites (PhosPhat 4.0; Heazlewood et al., 2008) and a total of 271 phosphopeptides on 226 proteins ( $26 \%$ of all quantified phosphopeptides) significantly changed in abundance (ANOVA $p$ value $\leq .05$ ) at either the D-L, L-D or both transitions (Table 1; Table S4).

\section{2 | Most proteins with diurnal changes in abundance fluctuate independently of their transcript levels and belong to specific functional networks}

To clarify which biological processes possess protein abundance dynamics, we grouped all significantly changing proteins with similar accumulation profiles into clusters and then subjected these clusters to GSEA. Not all clusters exhibited classic cosine dynamics, but instead have complex profiles at specific times of day. Each of the resulting clusters (CL1-CL6) is enriched for proteins involved in specific processes ( $p$ value $\leq .01$, gene set size $\geq 2$; Figure 1a,b; Data S1S6). Of these clusters, the CL3 abundance profile is especially complex with a sharp minimum during the second half of the light period that is also found at the transcript level for selected proteins in this group. CL3 is comprised of proteins involved in membrane related processes and ribosome biogenesis. Cluster CL4 is enriched for proteins involved in nitrogen metabolism and photosynthesis that have increasing levels during the first hours of the day, followed by a 
(a)

\section{Diurnal Protein Levels}

(b)

$$
\begin{gathered}
\text { Enrichment } \\
\text { Analysis }
\end{gathered}
$$

(c)

(d)

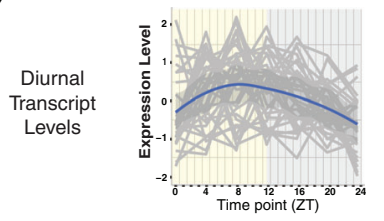
Subcellular
Localization

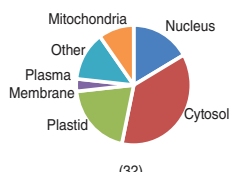

Cluster 2

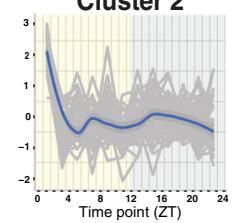

- Cellular response to gravity - Nitrogen metabolism - Cellular iron homeostasis

- ER-associated ubiquitin

-dependent protein catabolic process

Chloroplast stroma protein

impor
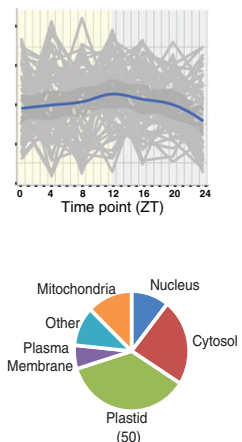

Cluster 3

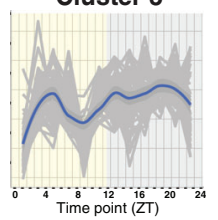

Mitochondria outer membrane - Ribosome biogenesis Anion channel activity Porin activity

Regulation of transmembrane transport
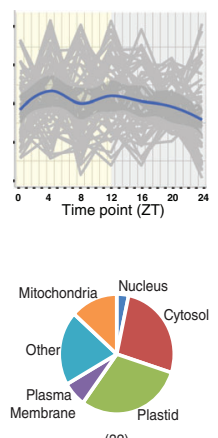

Cluster 4

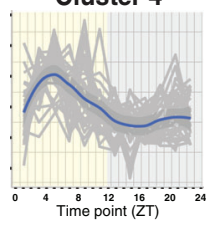

- Nitrate reductase activity - Photosynthesis - Response to wounding

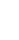

- Nuclear proteasome complex - Aerobic Respirame
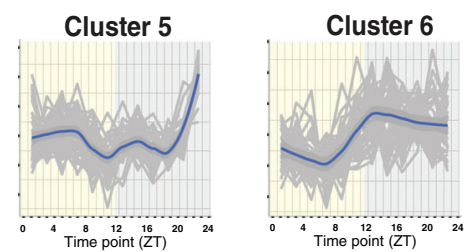

luster 6

- Quinone binding
- Isoquinoline alkaloid
biosynthesis

biosynthesis

Stachyose biosynthesis

RNA binding

ATP-dependent helicase activity
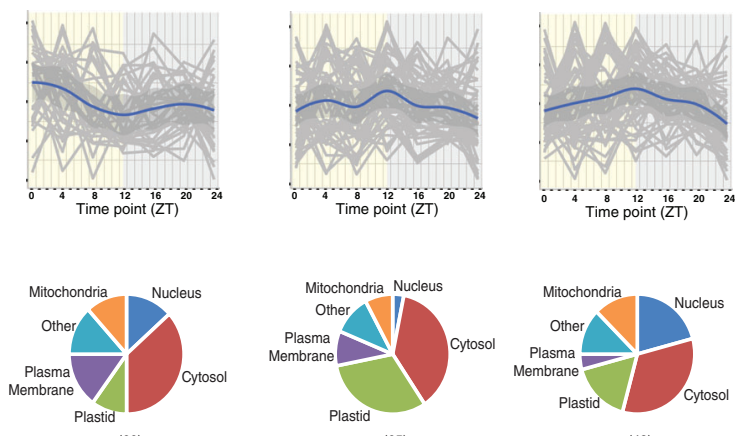

(49)

FIGURE 1 Analysis of the diurnal proteome: clustering, enrichment analysis and subcellular localization. (a) Significantly changing proteins ( $F C \geq 1.5$, ANOVA $p$ value $\leq .05, \geq 2$ peptides) were subjected to an unsupervised clustering analysis (GProX; http://gprox.sourceforge.net) resolving six protein clusters. $Y$ - and X-axis depict standardized expression level and harvest time (Zeitgeber time; ZT), respectively. Median expression is depicted in blue. (b) Term enrichment analysis of significantly changing proteins using SetRank ( $p$ value $\leq .01$, size $\geq 2$ ).

(c) Standardized diurnal transcript expression level of each corresponding clustered protein (Log10). Median expression is depicted in blue. Transcript expression level was obtained from Diurnal DB (http://diurnal.mocklerlab.org/). (d) In silico subcellular localization analysis of significantly changing proteins using SUBAcon (SUBA3; http://suba3.plantenergy.uwa.edu.au). Bracketed numbers represent the number of proteins per cluster

reduction until the end of the day, while levels are stable during night. CL6 exhibits a similar pattern as CL4, but seems to be shifted by 4-6 hr so that the peak protein levels peak at dusk. CL6 is enriched for proteins involved in metabolic and RNA-related processes that indicate a systemic change in the plant cell environment.

We compared the proteins in CL1-CL6 with their corresponding transcript expression profiles using transcriptome data generated from whole Arabidopsis rosettes grown and harvested in comparable conditions and at similar time-points (Figure 1c). This revealed that the dynamics of CL1 to CL6 protein changes are not strictly correlated with the diurnal abundance changes of their transcripts (Figure 1c; Data S1-S6), as has been found in other studies (Abraham et al., 2016; Baerenfaller et al., 2012; Graf et al., 2017; Seaton et al., 2018). We then determined the subcellular compartmentalization of proteins in each cluster using the consensus localization predictor SUBAcon (SUBA3; http://suba3.plantenergy.uwa.edu.au; Figure 1d) (Tanz et al., 2013). Most clusters exhibited a similar distribution of localizations with the exception of CL4, which had an expanded complement of cytosolic and plasma membrane proteins coupled with a decrease in plastid-targeted proteins.

To determine connections between proteins with changing abundances, we next built functional association networks for each cluster using STRING-DB (http://string-db.org; Figure 2) to estimate association confidence between protein nodes, while subcellular localization information was included to resolve colocalized nodes. This analysis strategy resolved multiple protein hubs within each cluster that have variable degrees of interconnectedness to the depicted biological processes, with some processes complementing those enriched by GSEA (Figure 1b). Proteins with no known connections above the set association threshold were removed from the network.

Using our STRING-DB analysis approach, we defined network structures for proteins belonging to: RNA splicing (CL1) and processing (CL6; RNA helicases and binding proteins), chloroplastrelated processes (CL4 and 5, light detection; CL1 and CL5, carbohydrate/starch metabolism; CL2, redox regulation), cell metabolism (CL4, nitrogen and fatty acid metabolism), secretion and intracellular transport (CL2), cell wall biosynthesis (CL5) as well as cytosolic (CL1, 3 and 5), mitochondrial (CL3) and plastid (CL4 and 5) protein translation (Figure 2). Taken together, our GSEA and association network analyses provide new process- and proteinlevel information for when (time of day), where (subcellular compartment) and how (cellular processes) plants operate over a $24 \mathrm{hr}$ photoperiod. 
TABLE 1 Proteome and phosphoproteome coverage

\begin{tabular}{|lll|} 
& Proteome & Phosphoproteome \\
\hline Protein IDs & 7060 & 1091 \\
\hline Peptide IDs & $\mathrm{n} / \mathrm{a}$ & 1776 \\
\hline Proteins quantified & 4762 & 725 \\
\hline Peptides quantified & $\mathrm{n} / \mathrm{a}$ & 1056 \\
\hline Sig. changing proteins & ${ }^{*} 288$ & ${ }^{* *} 226$ \\
\hline Sig. changing peptides & $\mathrm{n} / \mathrm{a}$ & ${ }^{* *} 271$ \\
\hline
\end{tabular}

Note: Summary of the identified, quantified and significantly changing diurnal proteins, phosphopeptides and phosphoproteins. Quantification confidence thresholds are shown for the proteome (proteins identified by $\geq 2$ proteotypic peptides) and the phosphoproteome (site probability score $\geq 0.8$ ) quantified in $\geq 3$ biological replicates for each time point of the diurnal cycle and for each of the three time-points at the L-D and D-L transitions. The significance thresholds are shown for the proteome (FC $\geq 1.5$; ANOVA $p$ value $\leq .05$ ) and the phosphoproteome (ANOVA $p$ value $\leq .05$ ). Application of proteome and phosphoproteome significance thresholds are denoted by a single $\left({ }^{*}\right)$ and double $\left({ }^{* *}\right)$ asterisks, respectively.

\subsection{The influence of the circadian clock on diurnal fluctuations of proteins is limited}

To determine if the significant changes we measured in the diurnal proteome could be controlled by the circadian clock, we next compared our data to a quantitative proteomics dataset acquired under free-running (continuous light) conditions (Krahmer et al., 2019). Our dataset of 4,762 quantified proteins contains 1800 of the 2038 proteins (88\%) reported by Krahmer et al. (2019), allowing us to directly compare proteome results between studies (Data S7). To avoid identification of differences based on the fact that the quantitative proteome analysis described above and the JTK_cycle analysis used by Krahmer et al. (2019) differ in their methods, we also performed a JTK_cycle analysis to identify proteins cycling with a 22 or $24 \mathrm{hr}$ period (Hughes et al., 2010). Unlike our previous analysis of diurnal proteome fluctuations, which identified 288 significantly changing proteins regardless of cycling preconditions, the JTK_cycle analysis approach aims to elucidate proteins exhibiting diurnal fluctuations in the form of a cosine function, and correspondingly evaluates how well these changes in abundance fit with this expected cosine behaviour. JTK_cycle analysis estimates goodness of fit based on shuffling of protein values leading to a $p$ value. It then uses a Benjamini Hochberg correction to correct for multiple testing. In accordance with the analysis approach of Krahmer et al. (2019), we identified a total of 147 fluctuating proteins prior to multiple testing correction, which is comparable to the 211 found to fluctuate under continuous light conditions by Krahmer et al. (2019). Upon correcting for multiple testing, our JTK_cycle analysis revealed a total of 21 proteins to exhibit a significant fluctuation in abundance, of which three demonstrated a similar pattern under continuous light conditions (Figure 3a). Using the statistically relevant proteins only, our study and Krahmer et al. (2019) find three proteins to fluctuate in both studies, one only in L-D conditions and 7 only in continuous light. The fact that of these 11 proteins, only 10 have significant JTK-cycle fluctuations in continuous light (i.e., free-running condition) suggests that they are under circadian control, although additional proteome analysis of normal photoperiods prior to free-running conditions is needed to substantiate this possibility. Here, we find alpha-crystallin domain 32.1 (ACD32.1; AT1G06460) to fluctuate at the protein-level independent of the circadian clock. ACD32.1 was previously shown to be regulated diurnally at the transcript level in continuous light (Covington et al., 2008), but it did not fluctuate in the proteome data of Krahmer et al., 2019. ACD32.1 is a peroxisome-targeted chaperone protein (Pan et al., 2018) implicated in the suppression of protein aggregation (Ma, Haslbeck, Babujee, Jahn, \& Reumann, 2006). ACD32.1 peaks in abundance immediately after dark, suggesting a potential need for peroxisomal protein stability in the dark to maintain peroxisome functions required for plant growth, including fatty acid oxidation (Pan et al., 2018).

Given that the expression of many genes fluctuates at the transcript level, it is unexpected that such a low number of proteins exhibit rhythmic changes in protein abundance. For example, of the 22,641 diurnal gene expression profiles stored in the Diurnal Database v2.0 (http://diurnal.mocklerlab.org/), 40.6\% (9197) showed fluctuating transcripts in conditions that are comparable to ours (see Materials and Methods). Of our 4,762 proteins, we detected and quantified during the diurnal cycle, gene expression profiles for 4,468 proteins were also present in the Diurnal DB and the transcripts for 2,253 of these proteins had oscillating accumulation pattern. Of these oscillating transcripts, only $6.2 \%$ (140) encoded proteins that also showed peaks in abundance. For the remaining 2,215 transcripts that did not oscillate, $4.0 \%$ (88) still had proteins with peaks in their abundance, indicating that there is no stringent relationship between transcript oscillation and protein peak abundance.

To see if the fluctuating proteins that we did find are potentially explained by fluctuating transcripts, we searched the diurnal DB for the genes encoding the 21 proteins showing a significant JTK_cycle change in protein abundance (Figure 3a, magenta and blue) and found 18 of the genes. Of these 18, 15 were identified to possess diurnal changes in transcript abundance. For these 15 transcript-protein pairs, neither the protein nor the corresponding transcript levels were peaking at a specific Zeitgeber time (Figure 3b) and thus, these genes are likely regulated independently of each other. However, when comparing the patterns of individual pairs, normalizing for the transcript peak time, there was a median delay of $5.5 \mathrm{hr}$ between the peak transcript and peak protein (Figure 3c,d). Since such a shifted dependency of transcript and protein expression pattern is rare in our proteome dataset, its biological significance needs to be investigated further.

Together, while our diurnal proteome analysis revealed 288 proteins in different clusters that change in their abundance at different time intervals during the diurnal cycle, proteins for which their abundance changes follow a cosine function seem to be few when measured across the whole Arabidopsis rosette. The identification of only a single highly significant JTK-cycling protein in our diurnal proteome dataset is unexpected, but it is consistent with the limited fluctuations of proteins reported for measured proteomes of Arabidopsis 


\section{Cluster 1}

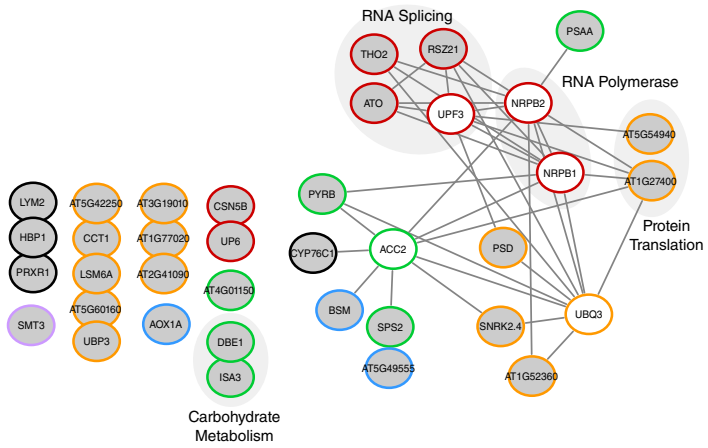

Cluster 3
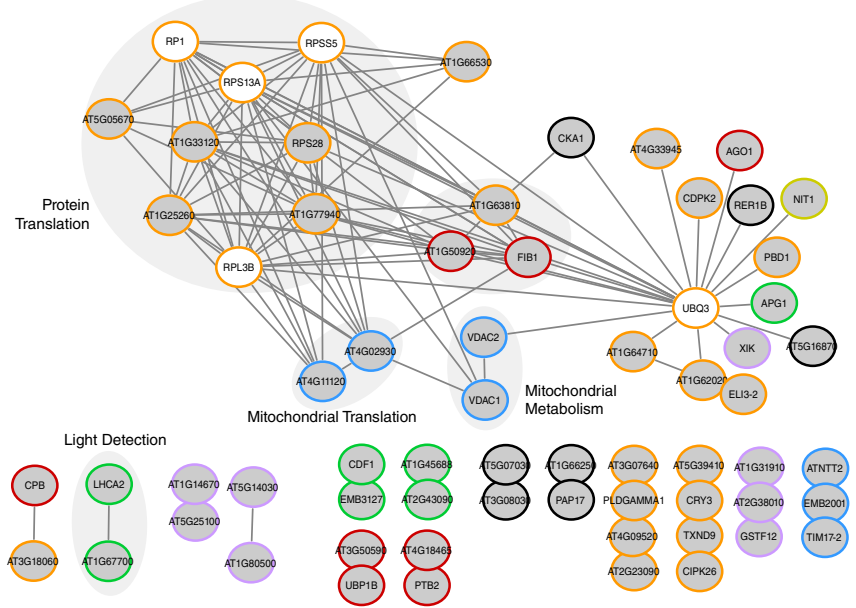

\section{Cluster 5}

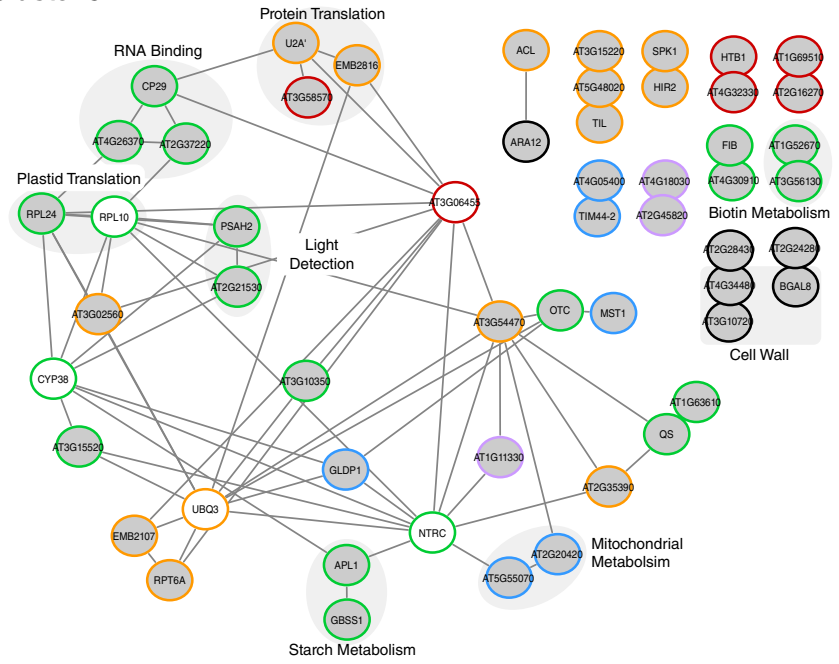

Cluster 2

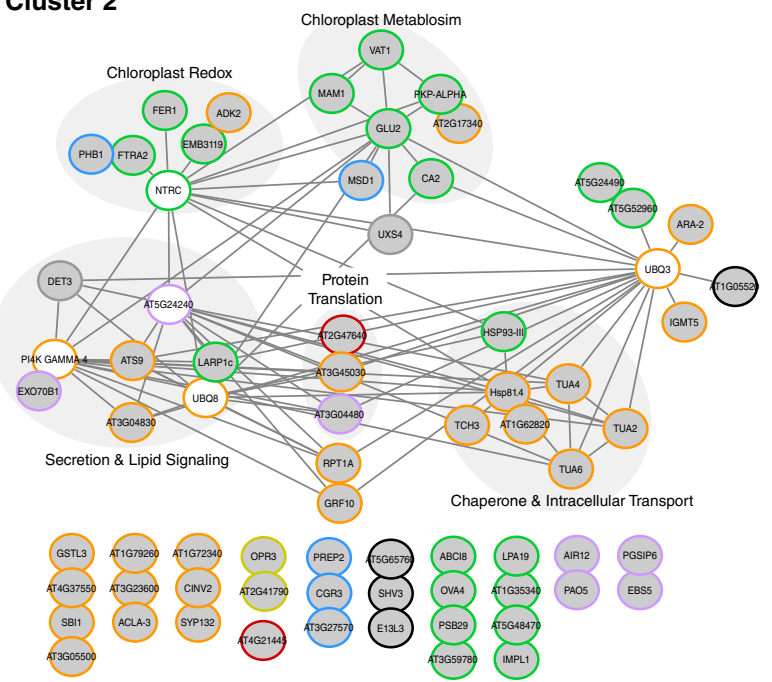

Cluster 4
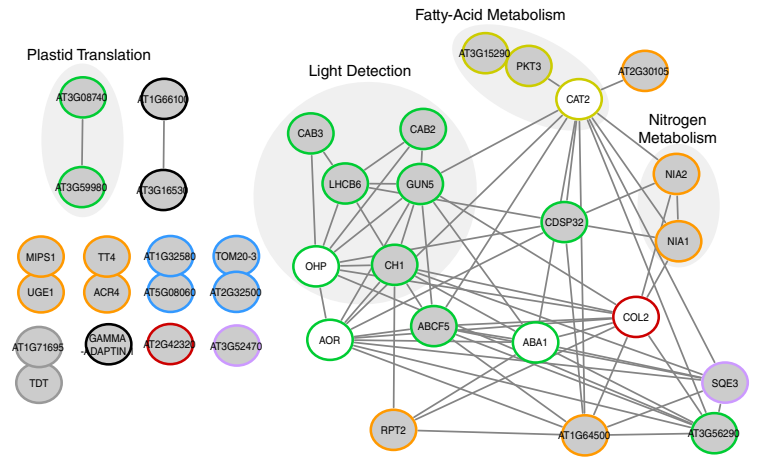

Cluster 6
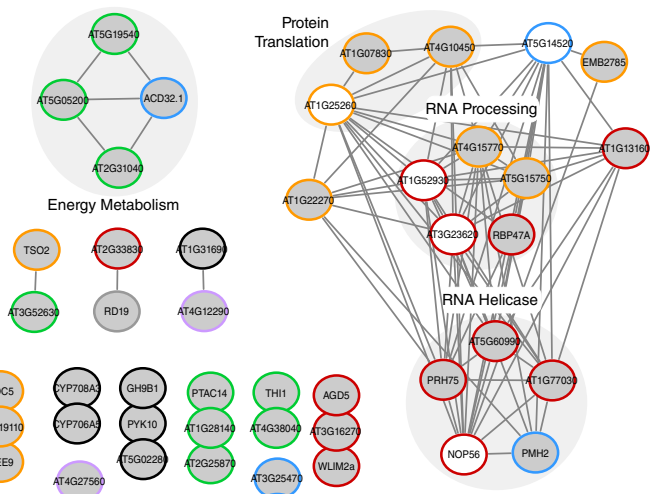
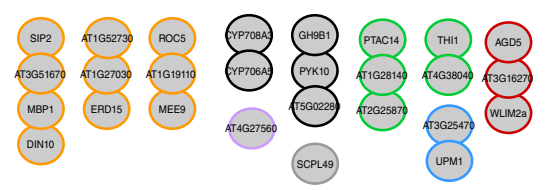

FIGURE 2 Interaction networks of the diurnal proteome. An association network analysis using STRING-DB (https://string-db.org/) of statistically significant diurnally changing proteins was performed using the generated unsupervised clusters shown in Figure 1 . Edge thickness indicates confidence of the connection between two nodes (0.5-1.0). Changing proteins (grey circles) are labelled by either their primary gene annotation or Arabidopsis gene identifier (AGI). The coloured outline of each node represents the in silico predicted subcellular localization of this protein (SUBAcon; suba3.plantenergy.uwa.edu.au). Nucleus (red), cytosol (orange), plastid (green), mitochondria (blue), plasma membrane (purple), peroxisome (dark yellow), endoplasmic reticulum/golgi/secreted (black) are depicted. A second layer of STRING-DB identified proteins (white nodes) not found in each respective significantly changing protein cluster was used to highlight the interconnectedness of proteins in the cluster. Multiple nodes encompassed by a labelled grey circle represent proteins involved in the same cellular process [Colour figure can be viewed at wileyonlinelibrary.com] 
(a)

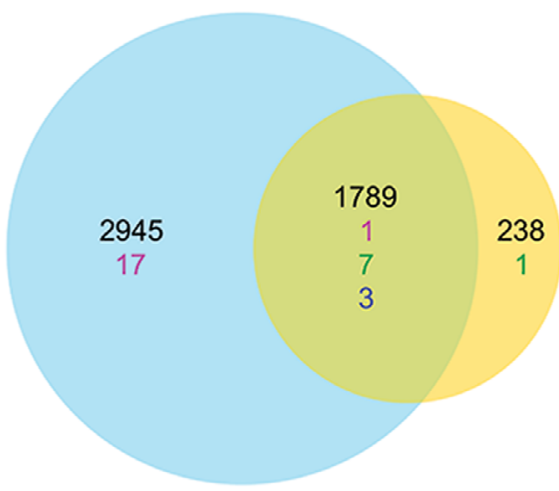

(b)

\begin{tabular}{|ccc|}
\hline Gene id & Protein Peak $[\mathrm{h}]$ & Transcript Peak $[\mathrm{h}]$ \\
\hline AT1G06460 & 13 & 9.5 \\
\hline AT1G25260 & 15 & \\
\hline AT1G27030 & 14 & \\
\hline AT1G29910 & 7 & \\
\hline AT1G32900 & 0 & \\
\hline AT1G44446 & 8 & 2.5 \\
\hline AT3G57520 & 4 & 23 \\
\hline AT1G64500 & 7 & 23.25 \\
\hline AT2G30520 & 20 & 12 \\
\hline AT2G31040 & 18 & 14 \\
\hline AT2G41430 & 13 & 11.5 \\
\hline AT3G27060 & 16 & 20 \\
\hline AT4G37760 & 8 & 1.5 \\
\hline AT4G39800 & 6 & \\
\hline AT5G13630 & 6 & 2.5 \\
\hline AT5G20250 & 18 & 22.5 \\
\hline AT5G40850 & 20 & 19.5 \\
\hline AT5G54770 & 18 & 14 \\
\hline AT5G54940 & 13 & 13 \\
\hline AT5G64840 & 7 & 0.5 \\
\hline AT5G64940 & 7 & 7 \\
\hline
\end{tabular}

(c)

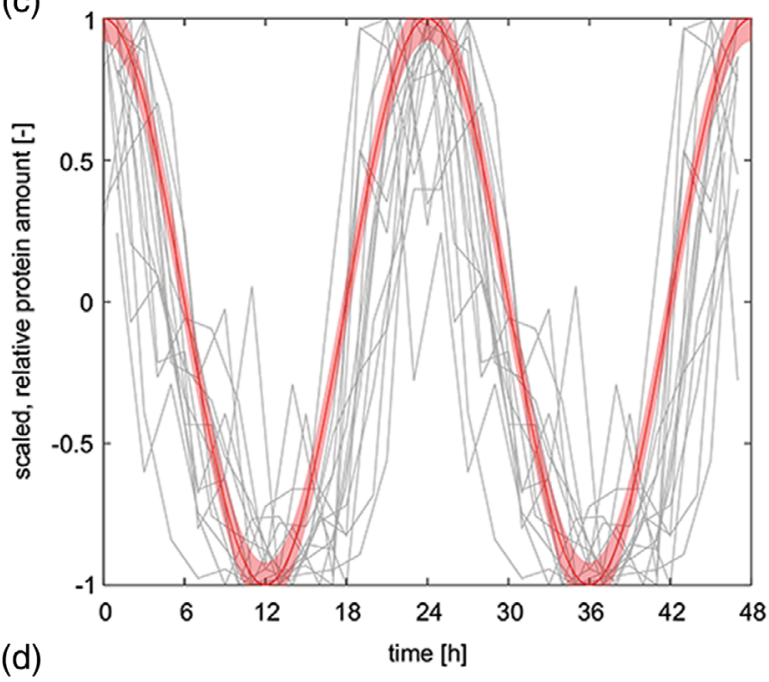

(d)

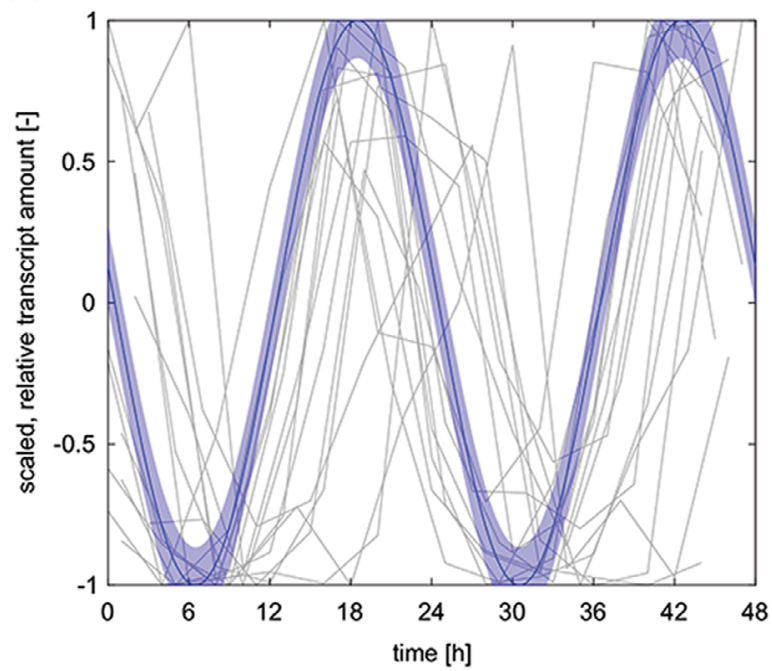

FIG URE 3 Comparative analysis of diurnal proteome to free-running circadian proteome (Krahmer et al., 2019). (a) Number of proteins measured in this study (blue circle) and Krahmer et al. (2019) (orange circle). Number of stable proteins (black), fluctuating proteins in our study only (magenta), Krahmer et al. (2019) only (green) and both studies (blue). (b) Table of 21 proteins that show significant (B.Q) fluctuation using JTK with their respective peak time period for protein and transcript levels (Diurnal DB, http://diurnal.mocklerlab.org/). (c and d) Normalized (Median = 0, Amplitude of 2) protein levels of 15 proteins both fluctuating in protein and transcript levels (gray lines) shifted to peak at time zero for protein levels in (c) and transcript levels in (d). Protein data were plotted twice to visualize a $48 \mathrm{hr}$ timeframe. The theoretical cosine functions with associated $99 \%$ confidence interval for protein levels (c, red) and transcript levels (d, blue) are shifted by $5.5 \mathrm{hr}$ [Colour figure can be viewed at wileyonlinelibrary.com]

wild type and circadian clock mutants growing in free-running cycles of continuous light (Choudhary et al., 2015; Krahmer et al., 2019). This low number of cycling diurnal proteins could be a consequence of the stringency of the JTK_Cycle analysis, which only tests for periodical protein changes following a cosine function, similar to the oscillating fluctuations of a large number of mRNAs regulated by the circadian clock in Arabidopsis and animals (Doherty \& Kay, 2010). Thus, in Arabidopsis rosettes, the diurnal abundance of most measured proteins does not seem to be affected by the circadian clock or regulated in concert with oscillating mRNA levels, which has also been found in growing Arabidopsis leaves at fewer diurnal timepoints (Baerenfaller et al., 2012). Seedling proteins have turnover rates ranging from $\log _{2} k-4$ to -7 (Fan et al., 2016) and the median degradation rate of proteins in growing Arabidopsis leaves is $\sim 0.11 \mathrm{~d}^{-1}$, but several proteins involved in protein synthesis, metabolic processes or photosynthesis have high degradation rates ranging from 0.6 up to $2.0 \mathrm{~d}^{-1}$ ( $\mathrm{Li}$, Nelson, et al., 2017). Some of the fluctuating proteins in CL1-6 (Figure 1) that we identified in the diurnal proteome fall into categories of proteins with high degradation rates, including proteins in ribosome biogenesis in CL3 (Figure 1) that contribute to the replacement of the leaf cytosolic ribosome population (Salih, Duncan, $\mathrm{Li}$, Trosch, \& Millar, 2020). For these proteins, oscillating mRNAs could contribute to the translational regulation of their changing accumulation (Missra et al., 2015), also in case of the 15 mRNAs and proteins whose median peaks are shifted by $5.5 \mathrm{hr}$ (Figure 3c,d). This does not exclude that oscillating mRNAs also contribute to the regulation of non-fluctuating proteins if the degradation and synthesis rates of these proteins are changing during the diurnal cycle. In 
TAB LE 2 GSEA of significantly changing phosphoproteins at the D-L and L-D transition

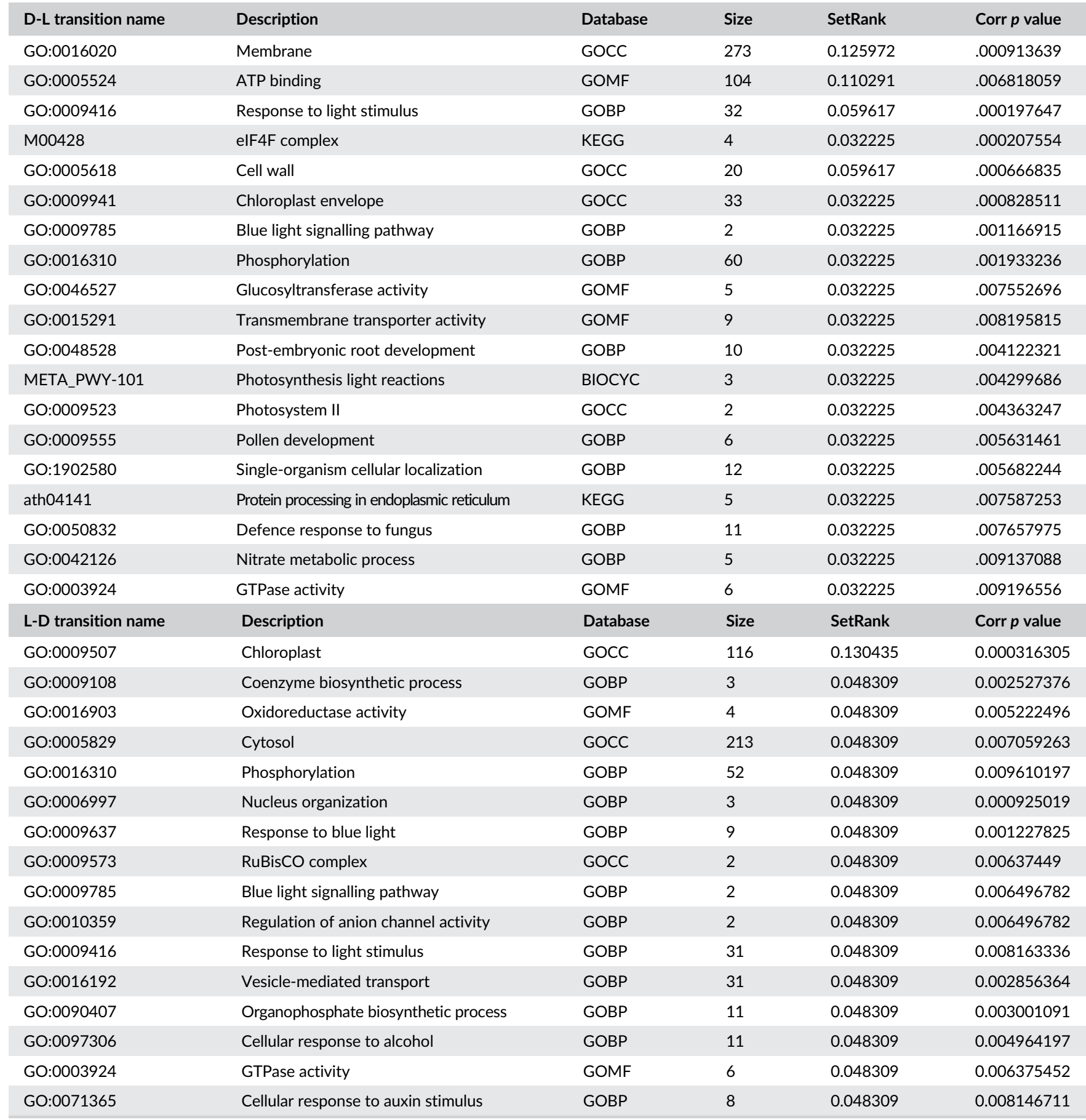

Note: GSEA was performed using SetRank ( $p$ value $\leq .01 ;$ FDR $\leq 0.05$, $\operatorname{minProt}=2$ ).

Arabidopsis, there is increasing evidence of diurnal and photoperiodic dynamics of mRNA translation (Mills, Enganti, \& von Arnim, 2018; Seaton et al., 2018). If dynamic regulation of protein degradation and synthesis is coupled to differential ribosomal loading of oscillating mRNAs, this would result in stable diurnal protein levels. At present, our diurnal proteome dataset cannot distinguish between these scenarios, but it establishes an important framework for investigating the role of protein degradation and synthesis in circadian and diurnal protein level regulation in more detail.

\subsection{Analysis of light-dark transitions in a diurnal cycle reveal dynamic fluctuation in the Arabidopsis phosphoproteome}

Protein phosphorylation is often associated with changing environmental conditions (Li, Li, et al., 2017; S. Zhang et al., 2019; Zhao et al., 2017). Therefore, we examined time-points before (30 min) and after (10 min, $30 \mathrm{~min}$ ) the D-L and L-D transitions for changes in the phosphoproteome (Figure S1). We found that 176 phosphopeptides 
(a) Dark-to-Light

(D-L)

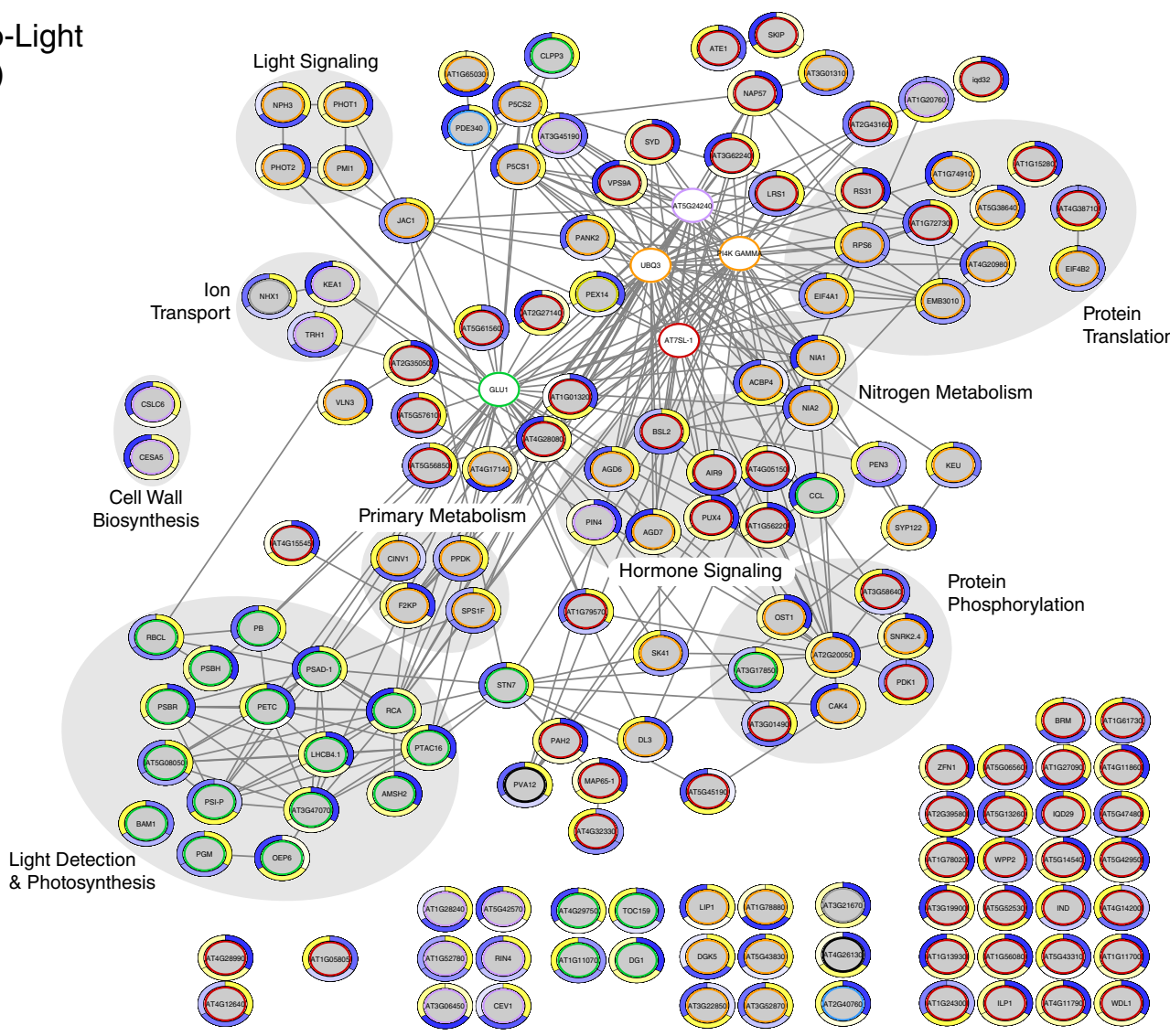

(b) Light-to-Dark

(L-D)

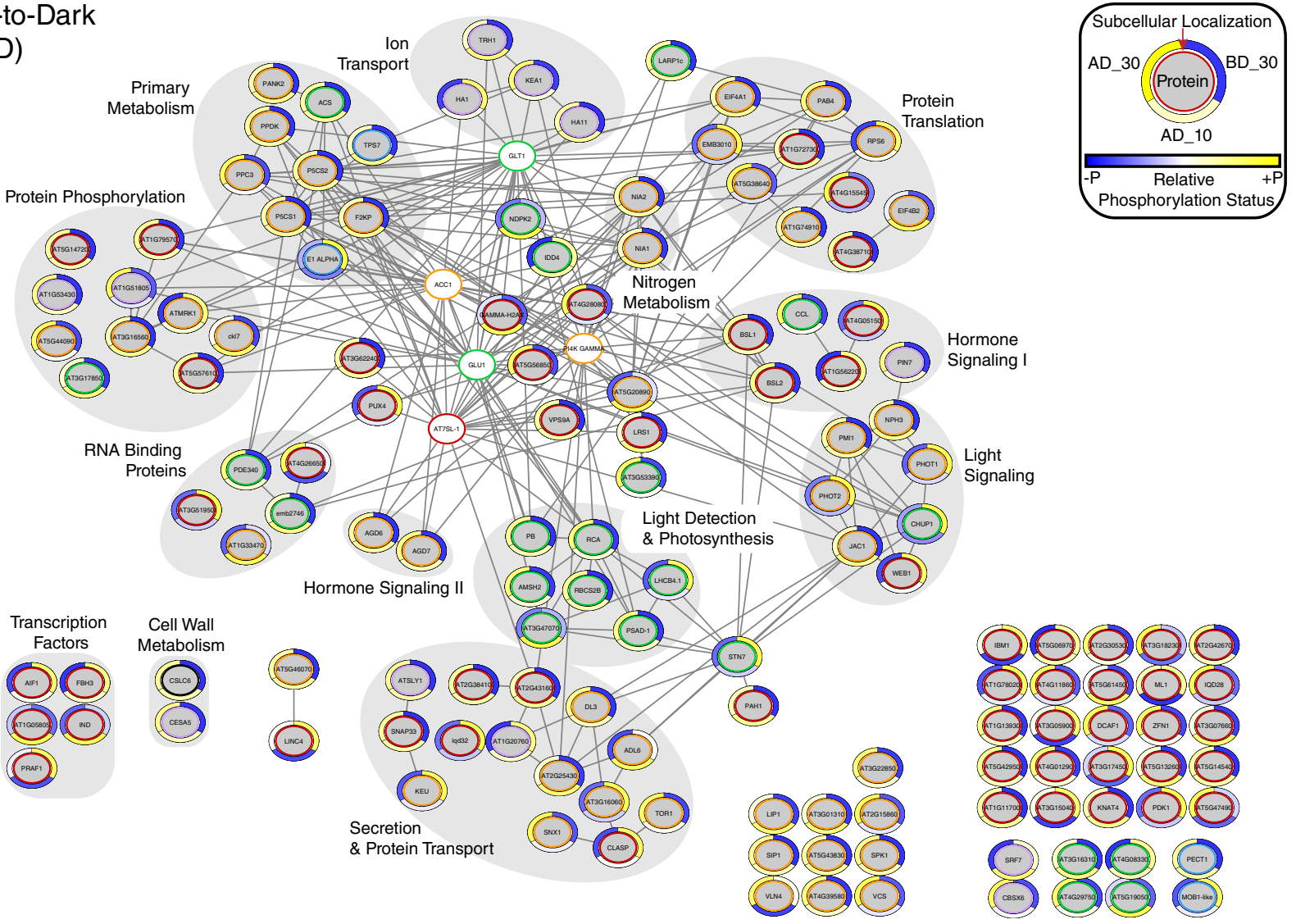

FIGURE 4 Legend on next page. 
from 153 proteins at the D-L transition and 164 phosphopeptides from 144 proteins at the L-D transition had significant changes in abundance (Figures S2 and S3; Table S4). We then benchmarked the quality of our dataset by querying it for proteins known to be diurnally regulated by protein phosphorylation (Table S5). This revealed phototropin 1 (PHOT1), phosphoenolpyruvate carboxylase (PEPC), nitrate reductase (NIA1 and NIA2) and CF1 ATP synthase. Phototropin 1 is phosphorylated in the light (Sullivan, Thomson, Kaiserli, \& Christie, 2009; Sullivan, Thomson, Lamont, Jones, \& Christie, 2008), while the NIA1, NIA2 and the CF1 ATP synthase beta-subunit are phosphorylated in the dark (Kanekatsu, Saito, Motohashi, \& Hisabori, 1998; Lillo, Meyer, Lea, Provan, \& Oltedal, 2004; G. Moorhead et al., 1999; Reiland et al., 2009). Quantitation of NIA1 and 2 protein phosphorylation changes across time-points revealed that NIA2 was more rapidly dephosphorylated on $\mathrm{Ser}^{534}$ at the D-L transition than NIA1, potentially relating to regulatory differences between NIA1 and 2. Additionally, we found a new NIA2 phosphorylation site at $\mathrm{Ser}^{63}$ with opposing diurnal changes in phosphorylation at the same transition (Figure S4). Both the rate of NIA1 and 2 phosphorylation as it relates to nitrate reduction and the new phosphorylation site require additional characterization that is beyond the scope of this study.

We next performed a GSEA of all significantly changing phosphoproteins ( $p$ value $\leq .01, F D R \leq 0.05$, gene set size $\geq 2$ ) at each transition. Enriched biological processes at the D-L transition include phosphoproteins involved in light detection, nitrogen metabolism, cell wallrelated processes and phosphorylation signalling, while phosphoproteins identified at the L-D transition are involved in light detection, vesicle-mediated transport, auxin signalling and nucleus organization (Table 2). We then generated a hierarchical heatmap of the phosphopeptides to identify clusters of proteins at each light transition with similar phosphorylation dynamics (Figures S2 and S3). When compared to datasets of phosphorylated proteins previously identified in Arabidopsis under free-running cycle conditions (Choudhary et al., 2015; Krahmer et al., 2019), or at the ED and EN time-points of a 12-hr photoperiod (Reiland et al., 2009; Uhrig et al., 2019), our data reveals new proteins that have diurnal changes in their phosphorylation status (Figures S2 and S3). Interestingly, parallel phosphorylation changes in L-D cluster I occur on proteins involved in nitrogen metabolism and the cell cycle. Nitrogen is acquired by plants primarily in the form of nitrate or ammonium, and is an essential macronutrient for plant growth. Nitrate signalling is linked to cell cycle progression through the teosinte branched $1 /$ cycloidea proliferating cell factor 20 (TCP20) - nin-like protein 6/7 (NLP6/7) regulatory network. TCP20 positively regulates genes encoding proteins involved in nitrate assimilation and signalling and down-regulates the expression of CYCB1;1, which encodes a key cell-cycle protein involved in the G2/M transition (Guan, 2017). Our data suggest that in addition to TCP20 transcriptional regulation, reversible protein phosphorylation may also play a role in this regulatory intersection between nitrate signalling and the cell cycle.

Similar to our analysis of protein abundance changes, we built association networks using STRING-DB to complement the GSEA analysis of the phosphoproteome (Figure 4). Most of the node clusters overlap between both the D-L (Figure 4a) and L-D (Figure 4b) networks, with larger clusters consisting of proteins involved in light detection and signalling, carbon and nitrogen metabolism, protein translation, hormone signalling, ion transport, cell wall related processes and protein phosphorylation. L-D transition-specific node clusters include RNA processing, transcription and secretion, and protein transport (Figure 4b). Similar to our proteome analyses, network association and GSEA analyses showed a high degree of overlap, indicating that the two approaches revealed the same cell processes in which proteins show differences in phosphorylation.

Given this, we hypothesize that the significantly changing Arabidopsis proteome measured over a $24 \mathrm{hr}$ photoperiod consists of proteins possessing key functions in each respective cellular process. As discussed above, protein abundance changes are generally not as widespread as transcriptome-level changes over a $24 \mathrm{hr}$ photoperiod (Baerenfaller et al., 2012; Graf et al., 2017; Seaton et al., 2018; Uhrig et al., 2019). Conversely, changes in protein phosphorylation can be dependent or independent of protein abundance fluctuations. To assess this, we compared the changing phosphoproteome and proteome and found that the majority of significantly changing phosphorylation events occur independent of protein abundance changes and therefore likely represent regulatory PTM events (Duby \& Boutry, 2009; Le, Browning, \& Gallie, 2000; Lillo et al., 2004; Muench, Zhang, \& Dahodwala, 2012). Based on these results, future investigations of which seemingly stable proteins/phosphoproteins and significantly changing phosphoproteins are in fact undergoing changes in their translation and turnover, but maintain their overall abundance (Li, Nelson, et al., 2017) are required to fully capture how the scale and dynamics of protein and PTM changes impact plant cell regulation.

FIGURE 4 Interaction networks of the diurnal phosphoproteome at the D-L and L-D transitions. An association network analysis of statistically significant diurnally changing phosphorylated proteins was performed using the STRING-DB (ANOVA $p$ value $\leq .05$ ). Edge thickness indicates strength of the connection between two nodes (0.5-1.0). Phosphorylated proteins (grey circles) are labelled by either their primary gene annotation or Arabidopsis gene identifier (AGI). Outer circle around each node depicts the standardized relative log2 FC in phosphorylation status of this protein between time-points. The sliding scale of yellow to blue represents a relative increase and decrease in phosphorylation, respectively. The inner coloured circles represent in silico predicted subcellular localization (SUBAcon; suba3.plantenergy.uwa.edu.au). Nucleus (red), cytosol (orange), plastid (green), mitochondria (blue), plasma membrane (purple), peroxisome (dark yellow), endoplasmic reticulum/golgi/ secreted (black) are depicted. A second shell of 5 STING-DB proteins (white circles) not found in our dataset was used to highlight the interconnectedness of the network. Multiple nodes encompassed by a labelled grey circle represent proteins involved in the same cellular process [Colour figure can be viewed at wileyonlinelibrary.com] 
(a)
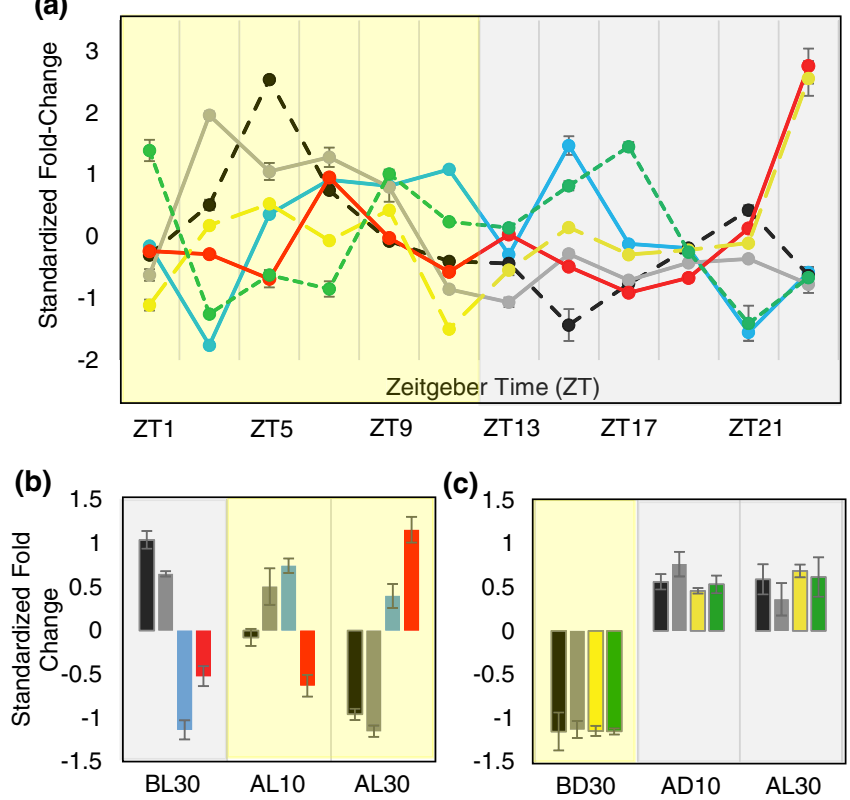

(c)

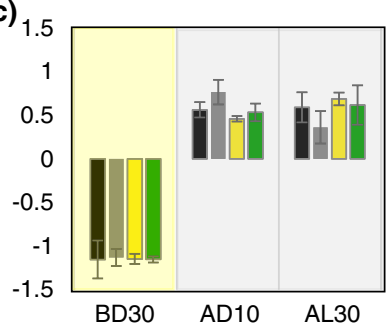

FIGURE 5 Proteins exhibiting a significant change in both diurnal protein abundance and protein phosphorylation status. Six proteins were found to significantly change in protein abundance and protein phosphorylation: AT1G10940 (SnRK2.4; blue), AT1G37130 (NIA2; black), AT1G77760 (NIA1; grey), AT4G32330 (TPX2; red), AT4G16340 (SPK1; yellow), AT4G35890 (LARP1c; green). (a) Diurnal protein abundance change profile. Standardized fold-change values are plotted relative to ZT. (b) D-L and (c) L-D phosphorylation change profiles. Standardized fold-change values are plotted relative to transition time-point either 10 or 30 min before light (BL), after light $(A L)$, before dark (BD) or after dark (AD). SE bars are shown [Colour figure can be viewed at wileyonlinelibrary.com]

\subsection{A small subset of the transition phosphoproteome has protein level changes}

Of the 226 proteins exhibiting a significant change in phosphorylation (Table 1), 60\% (136 proteins) were quantified in our proteome data (Table S6). These results are not unexpected because of the phosphopeptide enrichment strategy, and indicate that $40 \%$ of the phosphorylated proteins in our phosphoproteome dataset are of lower abundance and not among the 4,762 total quantified proteins. Further assessment of significantly changing phosphoproteins relative to the quantified proteome at the light transitions found that $25 \%$ (L-D) and $7.1 \%(D-L)$ of the changing phosphoproteins were not significantly changing at the protein level (TOST $p$ value $\leq .05, \varepsilon=0.4$ ).

We then directly compared the significantly changing phosphoproteome and proteome to identify proteins exhibiting a change in both diurnal protein abundance and phosphorylation status. We found that a total of six phosphoproteins ( $2.1 \%$ of all 288 proteins significantly changing in protein abundance; Table S6) that fit these criteria (Figure 5). These include nitrate reductase 1 (NIA1; AT1G77760) and 2 (NIA2; AT1G37130), PK SnRK2.4 (AT1G10940), Rho guanyl-nucleotide exchange factor SPK1 (AT4G16340), microtubule binding protein WDL5 (AT4G32330), and winged-helix DNA-

binding transcription factor family protein LARP1C (AT4G35890). NIA1 and 2 are directly related to nitrogen assimilation (Lillo, 2008; Lillo et al., 2004), while WDL5 has been implicated in mitigating ammonium toxicity through ethylene insensitive 3 (EIN3) (G. Li et al., 2019). SnRK2.4 binds fatty acid derived lipid phosphatidic acid to associate with the plasma membrane (Julkowska et al., 2015) and responds to changes in cell osmotic status (Munnik et al., 1999), while SPK1, WDL5 and LARP1C are connected to plant hormone signalling through abscisic acid (WDL5; Yu et al., 2019), jasmonic acid (LARP1C; B. Zhang, Jia, Yang, Yan, \& Han, 2012) and auxin (SPK1; Lin et al., 2012; M. Nakamura et al., 2018). Of these three proteins with concerted phosphorylation and abundance changes, only SPK1 showed a parallel increase in abundance and phosphorylation at the same transition (Figure 5), while WDL5 and LARP1C exhibited opposing patterns of phosphorylation and abundance changes, suggesting that phosphorylation may impact their turnover. Previously, proteins involved in phytohormone signalling have been found to be regulated by both protein phosphorylation and turnover (Dai et al., 2013; Qin et al., 2014), suggesting that these three proteins may represent new examples of hormone-mediated phosphodegrons or phosphoinhibited degrons (Vu, Gevaert, \& De Smet, 2018). Further examination of the ubiquitination status of these proteins and the proximity of those ubiquitin modifications to the annotated phosphorylation event are required to fully elucidate this hypothesis.

\section{6 | Motif analysis reveals diurnal utilization of phosphorylation sites}

We next hypothesized if we could connect our phosphoproteome data to a subset of PKs that may catalyse these diurnal events using a combination of motif enrichment analysis, published literature and available diurnal transcriptomic data as PKs are often below the limits of detection in proteomic studies. To understand which phosphorylation motifs are enriched in our dataset and to connect these to known PKs, we utilized Motif-X (motif-x.med.harvard.edu; Chou \& Schwartz, 2011; Schwartz \& Gygi, 2005). The significantly changing phosphorylated peptides at each transition were analysed against a background of all quantified phosphopeptides ( $p$ value $\leq .05$ ). Motifs corresponding to serine (pS) phosphorylation sites were enriched at each transition, while no enrichment of phosphorylated threonine (pT) or tyrosine (pY) motifs was found (Table S7). The lack of pY motif enrichment has also been reported when examining either ED vs EN (Reiland et al., 2009; Uhrig et al., 2019) or a free-running circadian cycle (Choudhary et al., 2015; Krahmer et al., 2019). Only one pT motif (pTP) has been previously associated with ED vs EN phosphoproteome changes (Uhrig et al., 2019). The lack of an enriched pTP motif here is likely due to our stringent multi-time point threshold requirement for each phosphorylation site to be considered for quantification. Furthermore, we would expect pS motifs to be enriched over either pT or pY motifs given that $\mathrm{pS}$ accounts for $84-86 \%$ of all phosphorylation events in plants, compared to only $10-12 \% \mathrm{pT}$ and 1-4\% pY (Mergner et al., 2020; Nakagami et al., 2010; Sugiyama et al., 2008). 
TAB LE 3 Proteins involved in plant cell processes with independent changes in abundance and/or phosphorylation.

\begin{tabular}{|c|c|c|c|c|}
\hline Biological process & AGI & Name & Description & $\begin{array}{l}\text { Abundance }(A) / \\
\text { Phosphorylation (P) }\end{array}$ \\
\hline \multirow[t]{16}{*}{ Translation } & AT5G54940 & elF1 & Translation initiation factor SUI1 family protein & A \\
\hline & AT1G27400 & RPL17A & Ribosomal protein L22p/L17e family protein & A \\
\hline & AT1G33120 & RPL9B & Ribosomal protein L6 family & A \\
\hline & AT3G45030 & RPS20A & Ribosomal protein $\mathrm{S} 10 \mathrm{p} / \mathrm{S} 20$ e family protein & A \\
\hline & AT5G64140 & RPS28C & Ribosomal protein S28 & A \\
\hline & AT3G02560 & RPS7B & Ribosomal protein S7e family protein & A \\
\hline & AT1G25260 & & Ribosomal protein L10 family protein & $A$ \\
\hline & AT3G08740 & chloroEF-P & Elongation factor $\mathrm{P}$ (EF-P) family protein & A \\
\hline & AT5G54600 & chloroL24 & Translation protein SH3-like family protein & A \\
\hline & AT1G13020 & elF4B & Eukaryotic initiation factor 4B2 & $\mathrm{P}$ \\
\hline & AT3G13920 & elF4A & Eukaryotic translation initiation factor $4 \mathrm{~A} 1$ & $\mathrm{P}$ \\
\hline & AT5G38640 & elF2Bc-d & NagB/RpiA/CoA transferase-like superfamily protein & $\mathrm{P}$ \\
\hline & AT3G13920 & elF4A & Eukaryotic translation initiation factor $4 \mathrm{~A} 1$ & $\mathrm{P}$ \\
\hline & AT4G20980 & elF3-d & Eukaryotic translation initiation factor 3 subunit 7 & $\mathrm{P}$ \\
\hline & AT4G31700 & RPS6A & Ribosomal protein $\mathrm{S} 6$ & $\mathrm{P}$ \\
\hline & AT5G10360 & RPS6B & Ribosomal protein S6e & $\mathrm{P}$ \\
\hline \multirow{3}{*}{ Primary metabolism } & AT2G42600 & PEPC2 & Phosphoenolpyruvate carboxylase 2 & $\mathrm{P}$ \\
\hline & AT3G14940 & PEPC3 & Phosphoenolpyruvate carboxylase 3 & $\mathrm{P}$ \\
\hline & AT5G20280 & SPS1F & Sucrose phosphate synthase $1 \mathrm{~F}$ & $\mathrm{P}$ \\
\hline \multirow[t]{4}{*}{ Carbohydrate metabolism } & AT1G32900 & GBSS1 & Granule bound starch synthase 1 & A \\
\hline & AT5G19220 & ADG2 & ADP glucose pyrophosphorylase & A \\
\hline & AT1G03310 & DBE1 & Debranching enzyme 1 & A \\
\hline & АТЗG23920 & BAM1 & Beta-amylase 1 & $P$ \\
\hline \multirow[t]{3}{*}{ Cell wall } & AT5G09870 & CESA5 & Cellulose synthase 5 & $P$ \\
\hline & АT3G07330 & CSLC6 & Cellulose-synthase-like C6 & $\mathrm{P}$ \\
\hline & AT2G18960 & HA1 & $\mathrm{H}^{+}$-ATPase 1 & $P$ \\
\hline
\end{tabular}

This makes it less likely to find an enrichment of pT and/or pY motifs in the phosphoproteome of plants. Of the phosphorylation sites (site probability score $\geq 0.8$ ), we quantified, $82.8,16.5$ and $0.7 \%$ were $\mathrm{pS}$, pT and pY, respectively, which aligns with previously reported distributions of phosphorylation events in $A$. thaliana (Mergner et al., 2020; Nakagami et al., 2010; Sugiyama et al., 2008).

At the L-D transition, we found 10 motifs corresponding to phosphorylation site motifs previously identified as targets of PKs CaMKII,
PAK1, extracellular signal-regulated kinase (ERK 1/2), proto-oncogene c-RAF (RAF1) and cell division cycle 2 (CDC2) PK A and B (Table S7). Six additional phosphorylation site motifs did not correspond to known kinase motifs, and therefore likely represent currently uncharacterized and possibly plant-specific motifs considering the large expansion of PKs in plants relative to humans (Lehti-Shiu \& Shiu, 2012; Zulawski et al., 2014). At the D-L transition, four of five identified motifs are known phosphorylation sites for checkpoint 
kinase 1 (CHK1), PAK2, calmodulin kinase IV (CaMKIV) and casein kinase (CKII) (Table S7). CKII is known to phosphorylate the core circadian clock transcription factors LHY and CCA1 (Lu et al., 2011), which also peak at the D-L transition (Kusakina \& Dodd, 2012).

The phosphoproteome data and motif analysis indicate that CAMKs are important mediators of L-D and D-L transition phosphosignalling and thus implicate the involvement of intracellular calcium $\left(\mathrm{Ca}^{2+}\right)$ in circadian regulation (Marti Ruiz et al., 2018). This suggests that calcium-dependent calmodulin (CaM) PK orthologs are interesting candidates for mediating circadian clock signalling. Unlike the enrichment of CKII motifs at only the D-L transition, we find enrichment of $\mathrm{Ca}^{2+}$ related kinases CaMKII (D-L and L-D) and CaMKIV (D-L) phosphorylation motifs at each transition (Table S7). Previous analyses have also identified $\mathrm{Ca}^{2+}$ kinase motifs enriched at both ED and EN (CDPK-like motifs; Uhrig et al., 2019). Additionally, SnRK1-related motifs were identified in the phosphoproteome data from Arabidopsis CCA1-Ox plants growing in a free-running cycle (Krahmer et al., 2019). SnRK1 is a central mediator of energy signalling between different organelles and also functions to phosphorylate CDPKs (Wurzinger, Nukarinen, Nagele, Weckwerth, \& Teige, 2018). Together, these data suggest a broader role for $\mathrm{Ca}^{2+}$ in diurnal plant cell regulation during the L-D and D-L transitions.

\section{7 | Key plant processes involve independent changes in both proteome and phosphoproteome}

When we queried the data for proteins that change in their abundance and/or phosphorylation status over the $24 \mathrm{hr}$ photoperiod, we found proteins predominantly involved in translation, cell wall biosynthesis and multiple aspects of plant metabolism. We hypothesize that these cellular processes are particularly susceptible to diurnal plant cell regulation at the protein level. The translation rates of Arabidopsis enzymes of light-induced metabolic reactions fluctuate diurnally and this correlates with their activity (Seaton et al., 2018). For example, several central metabolic enzymes are synthesized at 50\%-100\% higher rates during the light phase of the photoperiod ( $\mathrm{Pal}$ et al., 2013; Piques et al., 2009). Correspondingly, we identified 15 proteins involved in protein translation that have diurnal changes in abundance (Table 3; Table S2). Although they belong to several clusters shown in Figure 1a, nine of the proteins are grouped in CL3 with a general protein increase at the onset of light. In addition, we found eight translation-related proteins with changes in their phosphorylation status at $L-D$ and $D-L$ transitions, of which $5 / 8$ are eukaryotic initiation factor (eIF) proteins (Table 3; Table S8). Phosphorylation affects eukaryotic translation at the initiation step (Jackson, Hellen, \& Pestova, 2010; Le et al., 2000; Muench et al., 2012), and numerous elFs and ribosomal proteins show differences in phosphorylation levels between light and dark periods (BoexFontvieille et al., 2013; Enganti, Cho, Toperzer, Urquidi-Camacho, et al., 2018; Turkina, Klang Arstrand, \& Vener, 2011; Uhrig et al., 2019). Our analysis revealed additional diurnally regulated elFs and suggests that specific translational regulation mechanisms and ribosome composition could be controlled by light changes (e.g., day versus night).

We also find cell wall metabolic enzymes undergoing both diurnal fluctuations in protein abundance (Figure 2, Table 3; Table S2) and changes in phosphorylation status (Figure 3, Table 3; Table S4) at the D-L and L-D transitions. Cell wall biosynthesis is a major metabolic activity of growing plants (Barnes \& Anderson, 2017; Cosgrove, 2005). We find that cellulose synthase enzymes CESA5 (AT5G09870) and CSLC6 (AT3G07330) were rapidly phosphorylated at the L-D transition. CESA5 has been shown to be phosphorylated and phosphorylation memetic-mutant enzymes increase movement of the cellulose synthase complex (CSC) in dark-grown seedlings, indicating a photoperiod-dependent regulation cell wall biosynthesis (Bischoff et al., 2011). Diurnal cellulose synthesis may also be controlled by the intracellular trafficking of CSC enzymes as a result of changes in metabolism (Ivakov et al., 2017). In dark-grown hypocotyls the ratio of CESA5 to CESA6 phosphorylation in the CSC complex is important for cellulose synthesis (Bischoff et al., 2011). Our phosphoproteome results now provide additional information on the rate of CESA5 phosphorylation at the onset of that dark period. We also find phosphorylation of the plasma membrane $\mathrm{H}^{+}$-ATPase HA1 (AT2G18960) at the L-D transition (Figure 3b). Phosphorylation activates $\mathrm{H}^{+}$-ATPases (Duby \& Boutry, 2009; Sondergaard, Schulz, \& Palmgren, 2004) and implicates HA1 as a primary candidate $\mathrm{H}^{+}$ATPase in diurnal cell wall acidification to facilitate cell expansion during the night (Ivakov et al., 2017).

In addition to protein translation and cell wall-related processes, we identified a number of enzymes involved in lipid, carbohydrate and nitrogen metabolism that change at their protein levels (Figure 2, Table 3; Table S2) over the $24 \mathrm{hr}$ time-course or phosphorylation status at the D-L and L-D transitions (Table 3; Table S9). Several of these enzymes have been previously identified as being phosphorylated (PhosPhat 4.0) (Heazlewood et al., 2008); however, our sampling of three closely spaced time-points provides new information about the rate of protein phosphorylation changes at each transition. Moreover, our results demonstrate that in Arabidopsis metabolic enzymes are subject to changes in either protein abundance or phosphorylation, or both, which likely is of regulatory relevance for metabolic pathway flux.

Our data reveal that several enzymes related to fatty acid, biotin, mitochondrial acetyl-CoA and chloroplast metabolism have diurnal changes in abundance (Figure 2; Table 3; Table S2). Of particular interest are peroxisomal fatty acid $\beta$-oxidation enzymes 3-ketoacyl-CoA thiolase 2 (KAT2/PKT3; AT2G33150) and 3-hydroxyacyl-CoA dehydrogenase (MFP2/AIM1-like; AT3G15290). KAT2 is a central enzyme in peroxisomal fatty-acid degradation for the production of acetylCoA that is required for histone acetylation, which in turn affects DNA methylation (L. Wang et al., 2019), and ABA signalling (Jiang, Zhang, Wang, \& Zhang, 2011), which is essential to daily regulation of stomatal conductance. MFP2/AIM1-like is an uncharacterized ortholog of multifunctional protein 2 (MFP2) and enoyl-COA isomerase (AIM1), which are involved in indole-3-acetic acid and jasmonic acid metabolism (Arent, Christensen, Pye, Norgaard, \& Henriksen, 2010; 
Delker, Zolman, Miersch, \& Wasternack, 2007). KAT2 loss-of-function mutants require sucrose to supplement plant acetyl-CoA production, suggesting that diurnal changes in fatty acid degradation through KAT2 and MFP2/AIM1-like are possibly tied to sucrose production and that products downstream of KAT2 and MFP2/AIM1-like (e.g., hormones) are essential to plant growth and development (Pinfield-Wells et al., 2005). Previously, fatty acid and lipid metabolism in leaves and seedlings has been suggested to be diurnally/circadian clock regulated (Gibon et al., 2006; Hsiao et al., 2014; Kim, Nusinow, Sorkin, Pruneda-Paz, \& Wang, 2019; Y. Nakamura, 2018; Y. Nakamura et al., 2014). This includes diurnal changes in fatty acids and lipids (Gibon et al., 2006) in wild-type plants as well as diurnal changes in triacylglycerol (Hsiao et al., 2014) and phosphatidic acid (Kim et al., 2019) in the circadian clock double mutant Ihycca1. Complementing these studies, our findings provide a new protein-level understanding of when fatty acid and lipid metabolism is diurnally impacted that differs from our current transcript/metabolite-based knowledge, indicating that further protein-level investigations are required.

Furthermore, we also find diurnal changes in both protein abundance and protein phosphorylation for enzymes involved in carbohydrate metabolism (Table 3; Tables S2 and S4). Starch biosynthesis and degradation are diurnally regulated to manage the primary carbon stores in plants (Kotting, Kossmann, Zeeman, \& Lloyd, 2010). For example, granule bound starch synthase 1 (GBSS1; AT1G32900) levels increase preceding the D-L transition, likely in anticipation of starch granule formation (Szydlowski et al., 2011). Debranching enzyme 1 (DBE1, AT1G03310) increases in abundance at the end of the light period to facilitate effective starch degradation in the dark (Delatte, Trevisan, Parker, \& Zeeman, 2005). Other enzymes such as beta-amylase 1 (BAM1; AT3G23920) were phosphorylated immediately after the onset of light. Although the function of BAM1 phosphorylation is currently unknown, our results provide information to understand its regulation in stomatal starch degradation and sensitivity to osmotic changes in rosettes (Zanella et al., 2016).

Lastly, we identified enzymes in nitrogen metabolism that changed their phosphorylation status at the D-L and L-D transitions (Table 3; Figure S4; Table S9). This predominantly involved nitrate reductase 1 (NIA1; AT1G77760) and 2 (NIA2; AT1G37130) proteins. NIA1 and NIA2 are regulated both transcriptionally and posttranslationally by phosphorylation (Lillo, 2008; Lillo et al., 2004; P. Wang et al., 2011). Our results further the understanding of NIA regulation by newly defining a rate of change in the phosphorylation of these related isozymes at the L-D and D-L transitions, while also defining when peak NIA1 and NIA2 protein levels precisely occur relative to peak transcript levels (Table 3; Figure S4; Table S9). NIA1 and NIA2 maintain tissue-specific gene expression profiles, with NIA1 expression generally complementing that of NIA2 in the same organ. NIA1 was predominantly found in leaves, while NIA2 was predominantly found in meristematic tissue (Olas \& Wahl, 2019). We analysed whole Arabidopsis rosettes before bolting, of which developing leaves and apical meristematic tissue comprises only a small amount of total tissue. We therefore propose that the

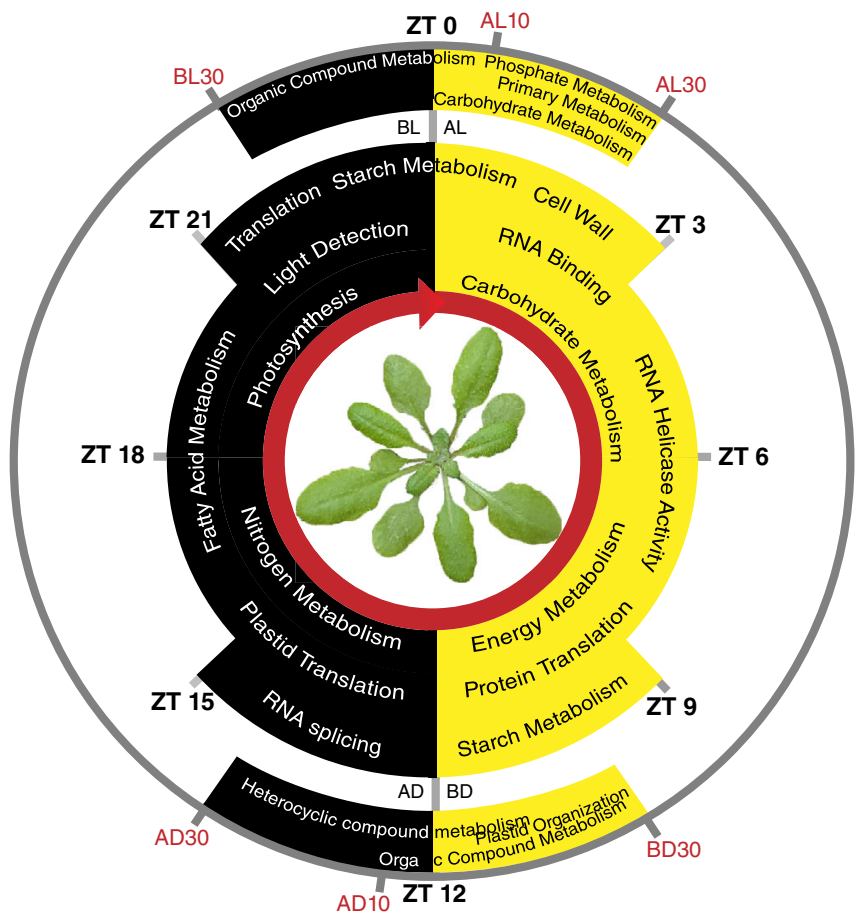

FIGURE 6 Schematic representation of Arabidopsis cellular and biological processes with diurnal fluctuations in protein abundance or protein phosphorylation. The inner three circles show terms of processes involving proteins with a maximal change in abundance during the day (yellow) or night (black). The outer circle shows terms of processes involving proteins with changes in protein phosphorylation at the dark-to-light (D-L) transition (top) or light-todark (L-D) transition (bottom). The segments of each inner circle relative to ZTO (day) or ZT12 (night) represent the approximate time interval in which proteins (ZT) and phosphoproteins (30 min before light or dark, 10 and 30 min after light or dark) involved in each process have their maximal change. The cellular and biological terms shown here were obtained by GO term enrichment of each protein and phosphoprotein cluster as outlined in Materials and Methods [Colour figure can be viewed at wileyonlinelibrary.com]

observed difference in NIA1 and NIA2 phosphorylation rates at these known regulatory phosphorylation sites reflects a higher sensitivity of NIA2 to changes in nitrate levels in meristematic and developing tissues (Olas et al., 2019).

Overall, our analysis of the phosphoproteome at three D-L and L-D time-points demonstrates the dynamism of phosphorylation events at both transitions. Plant genomes often encode multiple forms of enzymes (isozymes) in metabolic pathways, therefore knowing the temporal rate at which related co-expressed protein orthologs are modified by PTMs provides more detailed information about their cellular regulation. This information is particularly useful when deciding which protein isoform may be best for engineering increased pathway flux if two are present simultaneously. NIA1 and NIA2 represent good examples of how resolving differences in PTM rates helps to better understand the role of PTMs play in temporal protein regulation. Collectively, such insights will be broadly applicable to the other enzymes found in our dataset for more targeted experimentation. 


\section{4 | CONCLUSION}

To date, detailed analyses of plant functions during a $24 \mathrm{hr}$ diurnal cycle have predominantly focused on genome-wide changes in gene expression. Transcript-level changes can serve as a proxy for proteinlevel changes, but in plants, transcript levels often do not correlate with protein abundance. While proteomes have a narrower dynamic range than transcriptomes, they nevertheless complement transcriptome studies because they provide direct insights into proteinlevel changes. Our quantitative combined analysis of the proteome over a $12 \mathrm{hr}$ light:12 hr dark $24 \mathrm{hr}$ photoperiod and the phosphoproteome at the L-D and D-L transitions during the diurnal cycle in a single experimental workflow has generated new information on diurnal abundance fluctuations and/or phosphorylation changes for Arabidopsis proteins involved in different cellular and biological processes (Figure 6). In particular, understanding the specific functions of diurnally fluctuating ribosomal proteins involved in translation considering that hundreds of ribosomal protein isoforms are encoded by plant genomes with little information available to decipher their combinatorial assembly. Further elucidation of ribosome and protein translation regulation will be instrumental in filling the current knowledge gap between the transcriptome and proteome. Lastly, our phosphoproteome analysis during the transitions from D-L and L-D provides new information about candidate PKs catalysing diurnal phosphorylation events at each transition, providing new opportunities for future systems-level and targeted studies.

\section{ACKNOWLEDGMENTS}

This work was funded by TiMet - Linking the Clock to Metabolism (Grant Agreement 245143) supported by the European Commission (FP7-KBBE-2009-3). Experiments conducted at Forschungszentrum Jülich were partly funded by the Helmholtz Association. W.G. also acknowledges a Yushan Scholarship from the Ministry of Education (Taiwan) for his research.

\section{CONFLICT OF INTEREST}

The authors have no conflicts of interest.

\section{ORCID}

Wilhelm Gruissem (D) https://orcid.org/0000-0002-1872-2998

\section{REFERENCES}

Abraham, P. E., Yin, H., Borland, A. M., Weighill, D., Lim, S. D., De Paoli, H. C., ... Yang, X. (2016). Transcript, protein and metabolite temporal dynamics in the CAM plant Agave. Nature Plants, 2, 16178. https://doi.org/10.1038/nplants.2016.178

Adam, K., \& Hunter, T. (2018). Histidine kinases and the missing phosphoproteome from prokaryotes to eukaryotes. Laboratory Investigation, 98(2), 233-247. https://doi.org/10.1038/labinvest. 2017.118

Annunziata, M. G., Apelt, F., Carillo, P., Krause, U., Feil, R., Koehl, K., ... Stitt, M. (2018). Response of Arabidopsis primary metabolism and circadian clock to low night temperature in a natural light environment. Journal of Experimental Botany, 69(20), 4881-4895. https://doi.org/10. 1093/jxb/ery276
Arent, S., Christensen, C. E., Pye, V. E., Norgaard, A., \& Henriksen, A. (2010). The multifunctional protein in peroxisomal beta-oxidation: Structure and substrate specificity of the Arabidopsis thaliana protein MFP2. The Journal of Biological Chemistry, 285(31), 24066-24077. https://doi.org/10.1074/jbc.M110.106005

Baerenfaller, K., Massonnet, C., Walsh, S., Baginsky, S., Buhlmann, P., Hennig, L., ... Gruissem, W. (2012). Systems-based analysis of Arabidopsis leaf growth reveals adaptation to water deficit. Molecular Systems Biology, 8, 606. https://doi.org/10.1038/msb.2012.39

Barboza-Barquero, L., Nagel, K. A., Jansen, M., Klasen, J. R., Kastenholz, B., Braun, S., ... Fiorani, F. (2015). Phenotype of Arabidopsis thaliana semidwarfs with deep roots and high growth rates under water-limiting conditions is independent of the GA5 loss-of-function alleles. Annals of Botany, 116(3), 321-331. https://doi.org/10.1093/aob/mcv099

Barnes, W. J., \& Anderson, C. T. (2017). Release, recycle, rebuild: Cell wall remodeling, autodegradation, and sugar salvage for new wall biosynthesis during plant development. Molecular Plant, 11(1), 31-46. https://doi.org/10.1016/j.molp.2017.08.011.

Bischoff, V., Desprez, T., Mouille, G., Vernhettes, S., Gonneau, M., \& Hofte, H. (2011). Phytochrome regulation of cellulose synthesis in Arabidopsis. Current Biology, 21(21), 1822-1827. https://doi.org/10. 1016/j.cub.2011.09.026

Blasing, O. E., Gibon, Y., Gunther, M., Hohne, M., Morcuende, R., Osuna, D., ... Stitt, M. (2005). Sugars and circadian regulation make major contributions to the global regulation of diurnal gene expression in Arabidopsis. Plant Cell, 17(12), 3257-3281. https://doi.org/10. 1105/tpc.105.035261

Boersema, P. J., Raijmakers, R., Lemeer, S., Mohammed, S., \& Heck, A. J. (2009). Multiplex peptide stable isotope dimethyl labeling for quantitative proteomics. Nature Protocols, 4(4), 484-494. https://doi.org/10. 1038/nprot.2009.21

Boex-Fontvieille, E., Daventure, M., Jossier, M., Zivy, M., Hodges, M., \& Tcherkez, G. (2013). Photosynthetic control of Arabidopsis leaf cytoplasmic translation initiation by protein phosphorylation. PLoS One, 8 (7), e70692. https://doi.org/10.1371/journal.pone.0070692

Bradford, M. M. (1976). A rapid and sensitive method for the quantitation of microgram quantities of protein utilizing the principle of proteindye binding. Analytical Biochemistry, 72, 248-254.

Chou, M. F., \& Schwartz, D. (2011). Biological sequence motif discovery using motif-x. Current Protocols in Bioinformatics, 13(1), 15-24. https:// doi.org/10.1002/0471250953.bi1315s35

Choudhary, M. K., Nomura, Y., Wang, L., Nakagami, H., \& Somers, D. E. (2015). Quantitative circadian phosphoproteomic analysis of Arabidopsis reveals extensive clock control of key components in physiological, metabolic, and signaling pathways. Molecular \& Cellular Proteomics, 14(8), 2243-2260. https://doi.org/10.1074/mcp.M114. 047183

Cosgrove, D. J. (2005). Growth of the plant cell wall. Nature Reviews. Molecular Cell Biology, 6(11), 850-861. https://doi.org/10.1038/ nrm1746

Covington, M. F., Maloof, J. N., Straume, M., Kay, S. A., \& Harmer, S. L. (2008). Global transcriptome analysis reveals circadian regulation of key pathways in plant growth and development. Genome Biology, 9(8), R130. https://doi.org/10.1186/gb-2008-9-8-r130

Dai, M., Xue, Q., McCray, T., Margavage, K., Chen, F., Lee, J. H., ... Wang, H. (2013). The PP6 phosphatase regulates ABI5 phosphorylation and abscisic acid signaling in Arabidopsis. Plant Cell, 25(2), 517-534. https://doi.org/10.1105/tpc.112.105767

Delatte, T., Trevisan, M., Parker, M. L., \& Zeeman, S. C. (2005). Arabidopsis mutants Atisa1 and Atisa2 have identical phenotypes and lack the same multimeric isoamylase, which influences the branch point distribution of amylopectin during starch synthesis. The Plant Journal, 41(6), 815-830. https://doi.org/10.1111/j.1365-313X.2005.02348.x

Delker, C., Zolman, B. K., Miersch, O., \& Wasternack, C. (2007). Jasmonate biosynthesis in Arabidopsis thaliana requires peroxisomal beta- 
oxidation enzymes--additional proof by properties of pex6 and aim1. Phytochemistry, 68(12), 1642-1650. https://doi.org/10.1016/j. phytochem.2007.04.024

Doherty, C. J., \& Kay, S. A. (2010). Circadian control of global gene expression patterns. Annual Review of Genetics, 44, 419-444. https://doi.org/ 10.1146/annurev-genet-102209-163432

Duby, G., \& Boutry, M. (2009). The plant plasma membrane proton pump ATPase: A highly regulated P-type ATPase with multiple physiological roles. Pflügers Archiv, 457(3), 645-655. https://doi.org/10.1007/ s00424-008-0457-x

Enganti, R., Cho, S. K., Toperzer, J. D., Urquidi-Camacho, R. A., ... von Arnim, A. G. (2018). Phosphorylation of ribosomal protein RPS6 integrates light signals and circadian clock signal. Frontiers in Plant Science, 19(8), 2210-2227. https://doi.org/10.3389/fpls.2017.02210

Fan, K.-T., Rendahl, A. K., Chen, W.-P., Freund, D. M., Graym, W. M., Cohen, J. D., \& Hegeman, A. D. (2016). Proteome scale-protein turnover analysis using high resolution mass spectrometric data from stable-isotope labeled plants. Journal of Proteome Research, 15(3), 851-867. https://doi.org/10.1021/acs.jproteome.5b00772

Flis, A., Fernandez, A. P., Zielinski, T., Mengin, V., Sulpice, R., Stratford, K., ... Millar, A. J. (2015). Defining the robust behaviour of the plant clock gene circuit with absolute RNA timeseries and open infrastructure. Open Biology, 5(10), 150042. https://doi.org/10.1098/rsob.150042

Gibon, Y., Pyl, E. T., Sulpice, R., Lunn, J. E., Hohne, M., Gunther, M., \& Stitt, M. (2009). Adjustment of growth, starch turnover, protein content and central metabolism to a decrease of the carbon supply when Arabidopsis is grown in very short photoperiods. Plant Cell and Environment, 32(7), 859-874. https://doi.org/10.1111/j.1365-3040.2009. 01965.x

Gibon, Y., Usadel, B., Blaesing, O. E., Kamlage, B., Hoehne, M., Trethewey, R., \& Stitt, M. (2006). Integration of metabolite with transcript and enzyme activity profiling during diurnal cycles in Arabidopsis rosettes. Genome Biology, 7(8), R76. https://doi.org/10.1186/gb2006-7-8-R76

Graf, A., Coman, D., Uhrig, R. G., Walsh, S., Flis, A., Stitt, M., \& Gruissem, W. (2017). Parallel analysis of Arabidopsis circadian clock mutants reveals different scales of transcriptome and proteome regulation. Open Biology, 7(3), 160333. https://doi.org/10.1098/rsob. 160333

Grossmann, J., Roschitzki, B., Panse, C., Fortes, C., BarkowOesterreicher, S., Rutishauser, D., \& Schlapbach, R. (2010). Implementation and evaluation of relative and absolute quantification in shotgun proteomics with label-free methods. Journal of Proteomics, 73(9), 1740-1746. https://doi.org/10.1016/j.jprot.2010.05.011

Guan, P. (2017). Dancing with hormones: A current perspective of nitrate signaling and regulation in Arabidopsis. Frontiers in Plant Science, 8, 1697. https://doi.org/10.3389/fpls.2017.01697

Heazlewood, J. L., Durek, P., Hummel, J., Selbig, J., Weckwerth, W., Walther, D., \& Schulze, W. X. (2008). PhosPhAt: A database of phosphorylation sites in Arabidopsis thaliana and a plant-specific phosphorylation site predictor. Nucleic Acids Research, 36(Database issue), D1015-D1021. https://doi.org/10.1093/nar/gkm812

Hsiao, A. S., Haslam, R. P., Michaelson, L. V., Liao, P., Napier, J. A., \& Chye, M. L. (2014). Gene expression in plant lipid metabolism in Arabidopsis seedlings. PLoS One, 9(9), e107372. https://doi.org/10.1371/ journal.pone.0107372

Hughes, M. E., Hogenesch, J. B., \& Kornacker, K. (2010). JTK_CYCLE: An efficient nonparametric algorithm for detecting rhythmic components in genome-scale data sets. Journal of Biological Rhythms, 25(5), 372-380. https://doi.org/10.1177/0748730410379711

Ivakov, A., Flis, A., Apelt, F., Funfgeld, M., Scherer, U., Stitt, M., ... Suslov, D. (2017). Cellulose synthesis and cell expansion are regulated by different mechanisms in growing Arabidopsis hypocotyls. Plant Cell, 29(6), 1305-1315. https://doi.org/10.1105/tpc.16. 00782
Jackson, R. J., Hellen, C. U., \& Pestova, T. V. (2010). The mechanism of eukaryotic translation initiation and principles of its regulation. Nature Reviews. Molecular Cell Biology, 11(2), 113-127. https://doi.org/10. 1038/nrm2838

Jiang, T., Zhang, X. F., Wang, X. F., \& Zhang, D. P. (2011). Arabidopsis 3-ketoacyl-CoA thiolase-2 (KAT2), an enzyme of fatty acid beta-oxidation, is involved in ABA signal transduction. Plant \& Cell Physiology, 52 (3), 528-538. https://doi.org/10.1093/pcp/pcr008

Julkowska, M. M., McLoughlin, F., Galvan-Ampudia, C. S., Rankenberg, J. M., Kawa, D., Klimecka, M., ... Testerink, C. (2015). Identification and functional characterization of the Arabidopsis Snf1-related protein kinase SnRK2.4 phosphatidic acid-binding domain. Plant, Cell \& Environment, 38(3), 614-624. https://doi.org/10. 1111/pce.12421

Kanekatsu, M., Saito, H., Motohashi, K., \& Hisabori, T. (1998). The beta subunit of chloroplast ATP synthase (CFOCF1-ATPase) is phosphorylated by casein kinase II. Biochemistry and Molecular Biology International, 46(1), 99-105.

Kerk, D., Templeton, G., \& Moorhead, G. B. (2008). Evolutionary radiation pattern of novel protein phosphatases revealed by analysis of protein data from the completely sequenced genomes of humans, green algae, and higher plants. Plant Physiology, 146(2), 351-367. https://doi.org/ 10.1104/pp.107.111393

Kim, S. C., Nusinow, D. A., Sorkin, M. L., Pruneda-Paz, J., \& Wang, X. (2019). Interaction and regulation between lipid mediator phosphatidic acid and circadian clock regulators. Plant Cell, 31(2), 399-416. https:// doi.org/10.1105/tpc.18.00675

Kotting, O., Kossmann, J., Zeeman, S. C., \& Lloyd, J. R. (2010). Regulation of starch metabolism: The age of enlightenment? Current Opinion in Plant Biology, 13(3), 321-329. https://doi.org/10.1016/j.pbi.2010. 01.003

Krahmer, J., Hindle, M., Perby, L., Nielson, T. H., VanOoijen, G., Halliday, K. J., ... Millar, A. J. (2019). Circadian protein regulation in the green lineage II. The clock gene circuit controls a phospho-dawn in Arabidopsis thaliana. bioRXiv. doi:

Kusakina, J., \& Dodd, A. N. (2012). Phosphorylation in the plant circadian system. Trends in Plant Science, 17(10), 575-583. https://doi.org/10. 1016/j.tplants.2012.06.008

Le, H., Browning, K. S., \& Gallie, D. R. (2000). The phosphorylation state of poly(A)-binding protein specifies its binding to poly(A) RNA and its interaction with eukaryotic initiation factor (elF) $4 \mathrm{~F}$, elFiso4F, and elF4B. The Journal of Biological Chemistry, 275(23), 17452-17462. https://doi.org/10.1074/jbc.M001186200

Lehti-Shiu, M. D., \& Shiu, S. H. (2012). Diversity, classification and function of the plant PK superfamily. Philosophical Transactions of the Royal Society of London. Series B, Biological Sciences, 367(1602), 2619-2639. https://doi.org/10.1098/rstb.2012.0003

Li, F., Li, M., Wang, P., Cox, K. L., Jr., Duan, L., Dever, J. K., ... He, P. (2017). Regulation of cotton (Gossypium hirsutum) drought responses by mitogen-activated protein (MAP) kinase cascade-mediated phosphorylation of GhWRKY59. The New Phytologist, 215(4), 1462-1475. https://doi.org/10.1111/nph.14680

Li, G., Zhang, L., Wang, M., Di, D., Kronzucker, H. J., \& Shi, W. (2019). The Arabidopsis AMOT1/EIN3 gene plays an important role in the amelioration of ammonium toxicity. Journal of Experimental Botany, 70(4), 1375-1388. https://doi.org/10.1093/jxb/ery457

Li, L., Nelson, C. J., Trosch, J., Castleden, I., Huang, S., \& Millar, A. H. (2017). Protein degradation rate in Arabidopsis thaliana leaf growth and development. Plant Cell, 29(2), 207-228. https://doi.org/10. $1105 /$ tpc. 16.00768

Lillo, C. (2008). Signalling cascades integrating light-enhanced nitrate metabolism. The Biochemical Journal, 415(1), 11-19. https://doi.org/ 10.1042/BJ20081115

Lillo, C., Meyer, C., Lea, U. S., Provan, F., \& Oltedal, S. (2004). Mechanism and importance of post-translational regulation of nitrate reductase. 
Journal of Experimental Botany, 55(401), 1275-1282. https://doi.org/ 10.1093/jxb/erh132

Lin, D., Nagawa, S., Chen, J., Cao, L., Chen, X., Xu, T., ... Yang, Z. (2012). A ROP GTPase-dependent auxin signaling pathway regulates the subcellular distribution of PIN2 in Arabidopsis roots. Current Biology, 22(14), 1319-1325. https://doi.org/10.1016/j.cub.2012.05.019

Lu, S. X., Liu, H., Knowles, S. M., Li, J., Ma, L., Tobin, E. M., \& Lin, C. (2011). A role for PK casein kinase2 alpha-subunits in the Arabidopsis circadian clock. Plant Physiology, 157(3), 1537-1545. https://doi.org/10. 1104/pp.111.179846

Ma, C., Haslbeck, M., Babujee, L., Jahn, O., \& Reumann, S. (2006). Identification and characterization of a stress-inducible and a constitutive small heat-shock protein targeted to the matrix of plant peroxisomes. Plant Physiology, 141(1), 47-60. https://doi.org/10.1104/pp.105. 073841

Manning, G., Whyte, D. B., Martinez, R., Hunter, T., \& Sudarsanam, S. (2002). The PK complement of the human genome. Science, 298 (5600), 1912-1934. https://doi.org/10.1126/science.1075762

Marti Ruiz, M. C., Hubbard, K. E., Gardner, M. J., Jung, H. J., Aubry, S., Hotta, C. T., ... Webb, A. A. R. (2018). Circadian oscillations of cytosolic free calcium regulate the Arabidopsis circadian clock. Nature Plants, 4(9), 690-698. https://doi.org/10.1038/s41477-0180224-8

Mergner, J., Frejno, M., List, M., Papacek, M., Chen, X., Chaudhary, A., ... Kuster, B. (2020). Mass-spectrometry-based draft of the Arabidopsis proteome. Nature, 579, 409-414. https://doi.org/10.1038/s41586020-2094-2

Mills, S. C., Enganti, R., \& von Arnim, A. G. (2018). What makes ribosomes tick? RNA Biology, 15(1), 44-54. https://doi.org/10.1080/15476286. 2017.1391444

Missra, M., Ernest, B., Lohoff, T., Jia, Q., Satterlee, J., Ke, K., \& von Arnim, A. G. (2015). The circadian clock modulates global daily cycles of mRNA ribosome loading. Plant Cell, 27(9), 2582-2599. https://doi. org/10.1105/tpc.15.00546

Mockler, T. C., Michael, T. P., Priest, H. D., Shen, R., Sullivan, C. M., Givan, S. A., ... Chory, J. (2007). The DIURNAL project: DIURNAL and circadian expression profiling, model-based pattern matching, and promoter analysis. Cold Spring Harbor Symposia on Quantitative Biology, 72, 353-363. https://doi.org/10.1101/sqb.2007.72.006

Moorhead, G., Douglas, P., Cotelle, V., Harthill, J., Morrice, N., Meek, S., ... MacKintosh, C. (1999). Phosphorylation-dependent interactions between enzymes of plant metabolism and 14-3-3 proteins. The Plant Journal, 18 (1), 1-12. https://doi.org/10.1046/j.1365-313x.1999.00417.x

Moorhead, G. B., Trinkle-Mulcahy, L., Nimick, M., De Wever, V., Campbell, D. G., Gourlay, R., ... Lamond, A. I. (2008). Displacement affinity chromatography of protein phosphatase one (PP1) complexes. BMC Biochemistry, 9, 28. https://doi.org/10.1186/1471-2091-9-28

Muench, D. G., Zhang, C., \& Dahodwala, M. (2012). Control of cytoplasmic translation in plants. WIREs RNA, 3(2), 178-194. https://doi.org/10. 1002/wrna.1104

Munnik, T., Ligterink, W., Meskiene, I. I., Calderini, O., Beyerly, J., Musgrave, A., \& Hirt, H. (1999). Distinct osmo-sensing PK pathways are involved in signalling moderate and severe hyper-osmotic stress. The Plant Journal, 20(4), 381-388. https://doi.org/10.1046/j.1365313x.1999.00610.x

Nakagami, H., Sugiyama, N., Mochida, K., Daudi, A., Yoshida, Y., Toyoda, T., ... Shirasu, K. (2010). Large-scale comparative phosphoproteomics identifies conserved phosphorylation sites in plants. Plant Physiology, 153(3), 1161-1174. https://doi.org/10.1104/pp.110. 157347

Nakamura, M., Claes, A. R., Grebe, T., Hermkes, R., Viotti, C., Ikeda, Y., \& Grebe, M. (2018). Auxin and ROP GTPase signaling of polar nuclear migration in root epidermal hair cells. Plant Physiology, 176(1), 378-391. https://doi.org/10.1104/pp.17.00713

Nakamura, Y. (2018). Membrane lipid oscillation: An emerging system of molecular dynamics in the plant membrane. Plant \& Cell Physiology, 59 (3), 441-447. https://doi.org/10.1093/pcp/pcy023

Nakamura, Y., Andres, F., Kanehara, K., Liu, Y. C., Coupland, G., \& Dormann, P. (2014). Diurnal and circadian expression profiles of glycerolipid biosynthetic genes in Arabidopsis. Plant Signaling \& Behavior, 9(9), e29715. https://doi.org/10.4161/psb.29715

Nohales, M. A., \& Kay, S. A. (2016). Molecular mechanisms at the core of the plant circadian oscillator. Nature Structural \& Molecular Biology, 23(12), 1061-1069. https://doi.org/10.1038/nsmb.3327

Oakenfull, R. J., \& Davis, S. J. (2017). Shining a light on the Arabidopsis circadian clock. Plant, Cell \& Environment, 40, 2571-2585. https://doi. org/10.1111/pce.13033

Olas, J. J., Van Dingenen, J., Abel, C., Dzialo, M. A., Feil, R., Krapp, A., ... Wahl, V. (2019). Nitrate acts at the Arabidopsis thaliana shoot apical meristem to regulate flowering time. The New Phytologist, 223(2), 814-827. https://doi.org/10.1111/nph.15812

Olas, J. J., \& Wahl, V. (2019). Tissue-specific NIA1 and NIA2 expression in Arabidopsis thaliana. Plant Signaling \& Behavior, 14(11), 1656035. https://doi.org/10.1080/15592324.2019.1656035

Olsen, J. V., Blagoev, B., Gnad, F., Macek, B., Kumar, C., Mortensen, P., \& Mann, M. (2006). Global, in vivo, and site-specific phosphorylation dynamics in signaling networks. Cell, 127(3), 635-648. https://doi.org/ 10.1016/j.cell.2006.09.026

Pal, S. K., Liput, M., Piques, M., Ishihara, H., Obata, T., Martins, M. C., ... Stitt, M. (2013). Diurnal changes of polysome loading track sucrose content in the rosette of wild-type arabidopsis and the starchless pgm mutant. Plant Physiology, 162(3), 1246-1265. https://doi.org/10. 1104/pp.112.212258

Pan, R., Reumann, S., Lisik, P., Tietz, S., Olsen, L. J., \& Hu, J. (2018). Proteome analysis of peroxisomes from dark-treated senescent Arabidopsis leaves. Journal of Integrative Plant Biology, 60(11), 1028-1050. https:// doi.org/10.1111/jipb.12670

Pinfield-Wells, H., Rylott, E. L., Gilday, A. D., Graham, S., Job, K., Larson, T. R., \& Graham, I. A. (2005). Sucrose rescues seedling establishment but not germination of Arabidopsis mutants disrupted in peroxisomal fatty acid catabolism. The Plant Journal, 43(6), 861-872. https://doi.org/10.1111/j.1365-313X.2005.02498.x

Piques, M., Schulze, W. X., Hohne, M., Usadel, B., Gibon, Y., Rohwer, J., \& Stitt, M. (2009). Ribosome and transcript copy numbers, polysome occupancy and enzyme dynamics in Arabidopsis. Molecular Systems Biology, 5, 314. https://doi.org/10.1038/msb.2009.68

Qin, Q., Wang, W., Guo, X., Yue, J., Huang, Y., Xu, X., ... Hou, S. (2014). Arabidopsis DELLA protein degradation is controlled by a type-one protein phosphatase, TOPP4. PLoS Genetics, 10(7), e1004464. https:// doi.org/10.1371/journal.pgen.1004464

Rao, R. S., Thelen, J. J., \& Miernyk, J. A. (2014). In silico analysis of protein Lys-N-acetylation in plants. Frontiers in Plant Science, 5, 381. https:// doi.org/10.3389/fpls.2014.00381

Reiland, S., Messerli, G., Baerenfaller, K., Gerrits, B., Endler, A., Grossmann, J., ... Baginsky, S. (2009). Large-scale Arabidopsis phosphoproteome profiling reveals novel chloroplast kinase substrates and phosphorylation networks. Plant Physiology, 150(2), 889-903. https:// doi.org/10.1104/pp.109.138677

Rigbolt, K. T., Vanselow, J. T., \& Blagoev, B. (2011). GProX, a user-friendly platform for bioinformatics analysis and visualization of quantitative proteomics data. Mol Cell Proteomics, 10(8), O110.007450. https://doi. org/10.1074/mcp.0110.007450

Robles, M. S., Humphrey, S. J., \& Mann, M. (2017). Phosphorylation is a central mechanism for circadian control of metabolism and physiology. 
Cell Metabolism, 25(1), 118-127. https://doi.org/10.1016/j.cmet. 2016.10.004

Salih, K.-J., Duncan, O., Li, L., Trosch, J., \& Millar, A. H. (2020). The composition and turnover of the Arabidopsis thaliana $80 \mathrm{~S}$ cytosolic ribosome. The Biochemical Journal, 477(16), 3019-3032. https://doi.org/10. 1042/BCJ20200385

Schwartz, D., \& Gygi, S. P. (2005). An iterative statistical approach to the identification of protein phosphorylation motifs from large-scale data sets. Nature Biotechnology, 23(11), 1391-1398. https://doi.org/10. 1038/nbt1146

Seaton, D. D., Graf, A., Baerenfaller, K., Stitt, M., Millar, A. J., \& Gruissem, W. (2018). Photoperiodic control of the Arabidopsis proteome reveals a translational coincidence mechanism. Molecular Systems Biology, 14(3), e7962. https://doi.org/10.15252/msb. 20177962

Seluzicki, A., Burko, Y., \& Chory, J. (2017). Dancing in the dark: Darkness as a signal in plants. Plant, Cell \& Environment, 40, 2487-2501. https:// doi.org/10.1111/pce.12900

Simillion, C., Liechti, R., Lischer, H. E., loannidis, V., \& Bruggmann, R. (2017). Avoiding the pitfalls of gene set enrichment analysis with SetRank. BMC Bioinformatics, 18(1), 151. https://doi.org/10.1186/ s12859-017-1571-6

Sondergaard, T. E., Schulz, A., \& Palmgren, M. G. (2004). Energization of transport processes in plants. Roles of the plasma membrane $\mathrm{H}$ +-ATPase. Plant Physiology, 136(1), 2475-2482. https://doi.org/10. 1104/pp.104.048231

Staiger, D., Shin, J., Johansson, M., \& Davis, S. J. (2013). The circadian clock goes genomic. Genome Biology, 14(6), 208. https://doi.org/10.1186/ gb-2013-14-6-208

Sugiyama, N., Nakagami, H., Mochida, K., Daudi, A., Tomita, M., Shirasu, K., \& Ishihama, Y. (2008). Large-scale phosphorylation mapping reveals the extent of tyrosine phosphorylation in Arabidopsis. Molecular Systems Biology, 4, 193. https://doi.org/10.1038/msb. 2008.32

Sullivan, S., Thomson, C. E., Kaiserli, E., \& Christie, J. M. (2009). Interaction specificity of Arabidopsis 14-3-3 proteins with phototropin receptor kinases. FEBS Letters, 583(13), 2187-2193. https://doi.org/10.1016/j. febslet.2009.06.011

Sullivan, S., Thomson, C. E., Lamont, D. J., Jones, M. A., \& Christie, J. M. (2008). In vivo phosphorylation site mapping and functional characterization of Arabidopsis phototropin 1. Molecular Plant, 1(1), 178-194. https://doi.org/10.1093/mp/ssm017

Szklarczyk, D., Morris, J. H., Cook, H., Kuhn, M., Wyder, S., Simonovic, M., ... von Mering, C. (2017). The STRING database in 2017: Qualitycontrolled protein-protein association networks, made broadly accessible. Nucleic Acids Research, 45(D1), D362-D368. https://doi.org/10. 1093/nar/gkw937

Szydlowski, N., Ragel, P., Hennen-Bierwagen, T. A., Planchot, V., Myers, A. M., Merida, A., ... Wattebled, F. (2011). Integrated functions among multiple starch synthases determine both amylopectin chain length and branch linkage location in Arabidopsis leaf starch. Journal of Experimental Botany, 62(13), 4547-4559. https://doi.org/10.1093/ jxb/err172

Tanz, S. K., Castleden, I., Hooper, C. M., Vacher, M., Small, I., \& Millar, H. A. (2013). SUBA3: A database for integrating experimentation and prediction to define the SUBcellular location of proteins in Arabidopsis. Nucleic Acids Research, 41(Database issue), D1185-D1191. https:// doi.org/10.1093/nar/gks1151

Türker, C., Akal, F., Joho, D., Panse, C., Barkow-Oesterreicher, S., Rehrauer, H., \& Schlapbach, R. (2010, March). B-Fabric: The Swiss Army Knife for life sciences. Paper presented at Proceedings of the EDBT 2010, 13th International Conference on Extending Database
Technology, Lausanne, Switzerland. Retrieved from https://doi.org/ 10.1145/1739041.1739135

Turkina, M. V., Klang Arstrand, H., \& Vener, A. V. (2011). Differential phosphorylation of ribosomal proteins in Arabidopsis thaliana plants during day and night. PLoS One, 6(12), e29307. https://doi.org/10.1371/ journal.pone.0029307

Uehara, T. N., Mizutani, Y., Kuwata, K., Hirota, T., Sato, A., Mizoi, J., ... Nakamichi, N. (2019). Casein kinase 1 family regulates PRR5 and TOC1 in the Arabidopsis circadian clock. Proceedings of the National Academy of Sciences of the United States of America, 116(23), 11528-11536. https://doi.org/10.1073/pnas.1903357116

Uhrig, R. G., Labandera, A. M., \& Moorhead, G. B. (2013). Arabidopsis PPP family of serine/threonine protein phosphatases: Many targets but few engines. Trends in Plant Science, 18(9), 505-513. https://doi.org/ 10.1016/j.tplants.2013.05.004

Uhrig, R. G., Schlapfer, P., Roschitzki, B., Hirsch-Hoffmann, M., \& Gruissem, W. (2019). Diurnal changes in concerted plant protein phosphorylation and acetylation in Arabidopsis organs and seedlings. The Plant Journal, 99(1), 176-194. https://doi.org/10.1111/tpj.14315

Usadel, B., Blasing, O. E., Gibon, Y., Retzlaff, K., Hohne, M., Gunther, M., \& Stitt, M. (2008). Global transcript levels respond to small changes of the carbon status during progressive exhaustion of carbohydrates in Arabidopsis rosettes. Plant Physiology, 146(4), 1834-1861. https://doi. org/10.1104/pp.107.115592

Vu, L. D., Gevaert, K., \& De Smet, I. (2018). Protein language: Posttranslational modifications talking to each other. Trends in Plant Science, 23(12), 1068-1080. https://doi.org/10.1016/j.tplants.2018.09.004

Wang, L., Wang, C., Liu, X., Cheng, J., Li, S., Zhu, J. K., \& Gong, Z. (2019). Peroxisomal beta-oxidation regulates histone acetylation and DNA methylation in Arabidopsis. Proceedings of the National Academy of Sciences of the United States of America, 116(21), 10576-10585. https:// doi.org/10.1073/pnas.1904143116

Wang, P., Du, Y., \& Song, C. P. (2011). Phosphorylation by MPK6: A conserved transcriptional modification mediates nitrate reductase activation and NO production? Plant Signaling \& Behavior, 6(6), 889-891. https://doi.org/10.4161/psb.6.6.15308

Wisniewski, J. R., Zougman, A., Nagaraj, N., \& Mann, M. (2009). Universal sample preparation method for proteome analysis. Nature Methods, 6(5), 359-362.

Wurzinger, B., Nukarinen, E., Nagele, T., Weckwerth, W., \& Teige, M. (2018). The SnRK1 kinase as central mediator of energy signaling between different organelles. Plant Physiology, 176(2), 1085-1094. https://doi.org/10.1104/pp.17.01404

Yu, Y., Wang, J., Li, S., Kakan, X., Zhou, Y., Miao, Y., ... Huang, R. (2019). Ascorbic acid integrates the antagonistic modulation of ethylene and abscisic acid in the accumulation of reactive oxygen species. Plant Physiology, 179(4), 1861-1875. https://doi.org/10.1104/pp.18.01250

Zanella, M., Borghi, G. L., Pirone, C., Thalmann, M., Pazmino, D., Costa, A., ... Sparla, F. (2016). $\beta$-amylase 1 (BAM1) degrades transitory starch to sustain proline biosynthesis during drought stress. Journal of Experimental Botany, 67(6), 1819-1826. https://doi.org/10.1093/jxb/ erv572

Zhang, B., Jia, J., Yang, M., Yan, C., \& Han, Y. (2012). Overexpression of a LAM domain containing RNA-binding protein LARP1c induces precocious leaf senescence in Arabidopsis. Molecules and Cells, 34(4), 367-374. https://doi.org/10.1007/s10059-012-0111-5

Zhang, S., Feng, M., Chen, W., Zhou, X., Lu, J., Wang, Y., ... Gao, J. (2019). In rose, transcription factor PTM balances growth and drought survival via PIP2;1 aquaporin. Nature Plants, 5(3), 290-299. https://doi.org/10. 1038/s41477-019-0376-1

Zhao, C., Wang, P., Si, T., Hsu, C. C., Wang, L., Zayed, O., ... Zhu, J. K. (2017). MAP kinase cascades regulate the cold response by 
modulating ICE1 protein stability. Developmental Cell, 43(5), 618-629 e615. https://doi.org/10.1016/j.devcel.2017.09.024

Zhou, H., Low, T. Y., Hennrich, M. L., van der Toorn, H., Schwend, T., Zou, H., ... Heck, A. J. (2011). Enhancing the identification of phosphopeptides from putative basophilic kinase substrates using Ti (IV) based IMAC enrichment. Molecular \& Cellular Proteomics, 10(10), M110.006452. https://doi.org/10.1074/mcp. M110.006452

Zulawski, M., Schulze, G., Braginets, R., Hartmann, S., \& Schulze, W. X. (2014). The Arabidopsis Kinome: Phylogeny and evolutionary insights into functional diversification. BMC Genomics, 15(1), 548-562. https:// doi.org/10.1186/1471-2164-15-548

\section{SUPPORTING INFORMATION}

Additional supporting information may be found online in the Supporting Information section at the end of this article.

How to cite this article: Uhrig RG, Echevarría-Zomeño S, Schlapfer $\mathrm{P}$, et al. Diurnal dynamics of the Arabidopsis rosette proteome and phosphoproteome. Plant Cell Environ. 2021;44:

821-841. https://doi.org/10.1111/pce.13969 